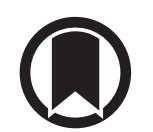

CrossMark

\title{
Phenotype characterisation of TBX4 mutation and deletion carriers with neonatal and paediatric pulmonary hypertension
}

\author{
Csaba Galambos (10)19, Mary P. Mullen ${ }^{2,19}$, Joseph T. Shieh ${ }^{3}$, \\ Nicolaus Schwerk ${ }^{4}$, Matthew J. Kielt ${ }^{5}$, Nicola Ullmann ${ }^{6}$, Renata Boldrini ${ }^{7}$, \\ Irena Stucin-Gantar ${ }^{8}$, Cristina Haass $^{9}$, Manish Bansal ${ }^{10}$, Pankaj B. Agrawal ${ }^{11}$, \\ Joyce Johnson ${ }^{12}$, Donatella Peca ${ }^{7}$, Cecilia Surace ${ }^{7}$, Renato Cutrera $\mathbb{1}^{6}$, \\ Michael W. Pauciulo ${ }^{13}$, William C. Nichols ${ }^{13}$, Matthias Griese ${ }^{14}$, Dunbar Ivy ${ }^{15}$, \\ Steven H. Abman ${ }^{16}$, Eric D. Austin ${ }^{5}$ and Olivier Danhaive ${ }^{17,18}$
}

@ERSpublications

TBX4 mutations and deletions are associated with abnormal distal lung development, persistent pulmonary hypertension of the newborn, paediatric pulmonary hypertension, multiple congenital anomalies and developmental disabilities http://bit.ly/2UXDrl3

Cite this article as: Galambos C, Mullen MP, Shieh JT, et al. Phenotype characterisation of TBX4 mutation and deletion carriers with neonatal and paediatric pulmonary hypertension. Eur Respir J 2019; 54: 1801965 [https://doi.org/10.1183/13993003.01965-2018].

ABSTRACT Rare variants in the T-box transcription factor 4 gene (TBX4) have recently been recognised as an emerging cause of paediatric pulmonary hypertension $(\mathrm{PH})$. Their pathophysiology and contribution to persistent pulmonary hypertension in neonates (PPHN) are unknown. We sought to define the spectrum of clinical manifestations and histopathology associated with TBX4 variants in neonates and children with $\mathrm{PH}$.

We assessed clinical data and lung tissue in 19 children with $\mathrm{PH}$, including PPHN, carrying TBX4 rare variants identified by next-generation sequencing and copy number variation arrays.

Variants included six 17q23 deletions encompassing the entire TBX4 locus and neighbouring genes, and 12 likely damaging mutations. 10 infants presented with neonatal hypoxic respiratory failure and PPHN, and were subsequently discharged home. PH was diagnosed later in infancy or childhood. Three children died and two required lung transplantation. Associated anomalies included patent ductus arteriosus, septal defects, foot anomalies and developmental disability, the latter with a higher prevalence in deletion carriers. Histology in seven infants showed abnormal distal lung development and pulmonary hypertensive remodelling.

TBX4 mutations and 17q23 deletions underlie a new form of developmental lung disease manifesting with severe, often biphasic $\mathrm{PH}$ at birth and/or later in infancy and childhood, often associated with skeletal anomalies, cardiac defects, neurodevelopmental disability and other anomalies. 


\section{Introduction}

Pulmonary arterial hypertension $(\mathrm{PAH})$ is a rare condition in infants and children, with a prevalence ranging between 4.8 and 8.1 cases per million [1-3], that leads to progressive right heart failure and high mortality despite recent progress in diagnosis and treatment [4]. PAH is a precapillary condition, and a subtype of pulmonary hypertension $(\mathrm{PH})$. Although $\mathrm{PAH}$ is heterogeneous, genetic defects relevant to pulmonary circulation underlie the majority of familial PAH cases and a significant subset of idiopathic PAH cases. Mutations in the bone morphogenetic protein (BMP) receptor type 2 gene (BMPR2) and other BMP-associated genes are found in $~ 70 \%$ of familial PAH cases, and $20 \%$ of idiopathic PAH cases in adults and children [5-8]. Recent studies have revealed a high prevalence of variants in T-box transcription factor 4 (TBX4), the gene associated with small patella syndrome (SPS) [9], in paediatric PAH [8, 10-12].

In the perinatal period, neonates may present with a form of $\mathrm{PH}$ known as persistent pulmonary hypertension of the newborn (PPHN), a condition with different underlying aetiologies causing persistent elevation of pulmonary vascular resistance and failure to transition from a fetal to postnatal circulatory pattern. PPHN is more common than paediatric $\mathrm{PAH}$, with an incidence of $0.18 \%, 20 \%$ of which is seemingly idiopathic [13]. Although PPHN is mostly reversible, with a mortality $<10 \%$, a small subset of cases typically unresponsive to therapy have developmental lung diseases [14]. Recently, TBX4 rare variants were described in three neonates with hypoxic respiratory failure caused by developmental lung disease $[15,16]$, expanding the spectrum of manifestations associated with these gene defects.

Given the potential importance of TBX4 expression during pulmonary development and the association between TBX4 and paediatric PH, we collected data from 19 paediatric patients with identified TBX4 variants and sought to more precisely determine the spectrum of manifestations in infants and children.

\section{Methods}

This series consists of cases selected from January 2014 to December 2017 from various clinical centres (supplementary table S1) on the basis of $\mathrm{PH}$ initially diagnosed by right heart catheterisation (RHC) in seven cases or echocardiography in 12 cases (table 1) during infancy or childhood and the presence of a TBX4 rare variant identified via clinical or research testing. Small nucleotide variants (SNVs) were identified by next-generation sequencing either from certified clinical laboratories or custom research panels, or by Sanger sequencing (supplementary table S1). For missense variants, the functional impact on protein structure was assessed by PolyPhen-2 (http://genetics.bwh.harvard.edu/pph2) and Combined Annotation Dependent Depletion v1.3 (CADD) [17]. Minor allele frequency $(<0.05)$ was checked searching the Exome Aggregation Consortium database [18]. Variants were compared to the ClinVar [19] and ClinGen [20] databases. Copy number variants (CNVs) were determined by chromosomal arrays. Variant significance was determined following the American College of Medical Genetics guidelines for CNVs [21] and SNVs [22]. De-identified patient data, including biometrics, family and neonatal history, initial and subsequent diagnostic studies and functional data, follow-up, and outcome, were extracted from registries or medical records (supplementary table S1). Clinically obtained lung tissue, when available, was re-analysed by a single pathologist (CG). This study was conducted in compliance with local institutional review boards.

Affiliations: ${ }^{1}$ Dept of Pathology and Laboratory Services, University of Colorado School of Medicine and Children's Hospital Colorado, Aurora, CO, USA. ${ }^{2}$ Dept of Cardiology, Boston Children's Hospital and Pediatrics Harvard Medical School, Boston, MA, USA. ${ }^{3}$ Institute for Human Genetics, Medical Genetics, University of California San Francisco, UCSF Benioff Children's Hospital, San Francisco, CA, USA. ${ }^{4}$ Clinic for Pediatric Pulmonology, Allergology and Neonatology, Hannover Medical School, Hannover, Germany. ${ }^{5}$ Division of Pediatric Pulmonology, Vanderbilt University Medical Center, Nashville, TN, USA. ${ }^{6}$ Division of Pediatric Pulmonology, Bambino Gesù Children's Hospital, Rome, Italy. ${ }^{7}$ Dept of Laboratory Medicine, Bambino Gesù Children's Hospital, Rome, Italy. ${ }^{8}$ Division of Perinatology, University Medical Centre, Ljubljana, Slovenia. ${ }^{9}$ Division of Neonatology, San Pietro-Fatebenefratelli Hospital, Rome, Italy. ${ }^{10}$ Division of Pediatric Cardiology, University of lowa Children's Hospital, lowa City, IA, USA. ${ }^{11}$ Division of Newborn Medicine and Genetics and Genomics, Harvard School of Medicine, Boston Children's Hospital, Boston, MA, USA. ${ }^{12}$ Dept of Pathology, Microbiology and Immunology, Vanderbilt University School of Medicine, Nashville, TN, USA. ${ }^{13}$ Division of Human Genetics, Cincinnati Children's Hospital Medical Center, Dept of Pediatrics, University of Cincinnati College of Medicine, Cincinnati, $\mathrm{OH}$, USA. ${ }^{14}$ Division of Pediatric Pulmonology, Ludwig-Maximilians-University, Munich, Germany. ${ }^{15}$ Division of Pediatric Cardiology, Dept of Pediatrics, University of Colorado School of Medicine and Children's Hospital Colorado, Aurora, CO, USA. ${ }^{16}$ Division of Pulmonary Medicine, Dept of Pediatrics, University of Colorado School of Medicine and Children's Hospital Colorado, Aurora, CO, USA. ${ }^{17}$ Division of Neonatology, University of California San Francisco Benioff Children's Hospital, San Francisco, CA, USA. ${ }^{18}$ Division of Neonatology, Catholic University of Louvain, Brussels, Belgium. ${ }^{19}$ Contributed equally to this work as joint first authors.

Correspondence: Olivier Danhaive, Division of Neonatology, Saint-Luc University Hospital, Catholic University of Louvain, 10 Avenue Hippocrate, Brussels B-1200, Belgium. E-mail: olivier.danhaiveđucsf.edu 


\begin{tabular}{|c|c|c|c|c|c|c|c|c|c|c|c|c|c|c|}
\hline \multirow[t]{2}{*}{ Case } & \multirow[t]{2}{*}{ Sex } & \multirow[t]{2}{*}{ Ethnicity } & \multicolumn{7}{|c|}{ Neonatal course } & \multicolumn{5}{|c|}{ Postnatal $\mathrm{PH}^{\#}$ course } \\
\hline & & & $\begin{array}{l}\text { GA weeks } \\
\text { BW g } \\
\text { Z-score }\end{array}$ & $\begin{array}{c}\text { Clinical } \\
\text { presentation }\end{array}$ & $\begin{array}{l}\text { ECMO } \\
\text { days }\end{array}$ & $\begin{array}{l}\text { iNO } \\
\text { days }\end{array}$ & $\begin{array}{l}\text { MV } \\
\text { days }\end{array}$ & $\begin{array}{l}\text { Home } \\
\text { days }\end{array}$ & Medication & & Initial diagnosis & $\begin{array}{l}\text { Tissue } \\
\text { sample }\end{array}$ & $\begin{array}{l}\text { Cardiovascular } \\
\text { medication }\end{array}$ & $\begin{array}{l}\text { Age at follow-up/ } \\
\text { death: outcome }\end{array}$ \\
\hline 1 & Male & Caucasian & $\begin{array}{c}36 \\
2000 \\
-1.70\end{array}$ & $\begin{array}{l}\text { PPHN" } \\
\text { RDS }\end{array}$ & $\begin{array}{l}\text { Yes } \\
12\end{array}$ & $\begin{array}{l}\text { Yes } \\
35\end{array}$ & $\begin{array}{l}\text { Yes } \\
45\end{array}$ & $\begin{array}{l}\text { Yes } \\
60\end{array}$ & $\begin{array}{c}\mathrm{O}_{2} \\
\text { PDE5i }\end{array}$ & $E$ & $\begin{array}{c}3 \text { months: } \\
\text { hypoxaemia } \\
4 \mathrm{~m} \text { : RSV } \\
\text { infection, HRF, } \\
\text { ECMO }\end{array}$ & $\begin{array}{l}4 \text { month } \\
\text { biopsy }\end{array}$ & $\mathrm{O}_{2}$, PDE5i & $\begin{array}{l}5 \text { months: death } \\
\text { from hypoxic } \\
\text { respiratory failure }\end{array}$ \\
\hline 2 & Male & Caucasian & $\begin{array}{c}38 \\
3090 \\
-0.15\end{array}$ & $\begin{array}{l}\text { Omphalocele } \\
\text { Transient } \\
\text { tachypnoea }\end{array}$ & No & No & Yes & $\begin{array}{l}\text { Yes } \\
17\end{array}$ & No & $E$ & $\begin{array}{c}5 \text { months: } \\
\text { hypoxaemia }\end{array}$ & - & $\mathrm{O}_{2}$ & $\begin{array}{l}4 \text { years: stable } \\
\text { mild PH, ILD }\end{array}$ \\
\hline 3 & Female & Caucasian & $\begin{array}{c}36 \\
2790 \\
0.42\end{array}$ & PPHN & No & $\begin{array}{c}\text { Yes } \\
6\end{array}$ & $\begin{array}{c}\text { Yes } \\
6\end{array}$ & $\begin{array}{l}\text { Yes } \\
41\end{array}$ & $\mathrm{O}_{2}$ & $\mathrm{E}$ & $\begin{array}{l}2 \text { months: } \\
\text { hypoxaemia }\end{array}$ & - & PDE5i, ERA & $\begin{array}{l}10 \text { years: stable } \\
\text { mild PH }\end{array}$ \\
\hline 4 & Female & & 40 & Normal & No & No & No & Yes & & C & $\begin{array}{c}4 \text { years: } \\
\text { intermittent } \\
\text { hypoxaemia } \\
5 \text { years: PDA } \\
\text { closure } \\
\text { Lack of } \\
\text { follow-up until } \\
\text { age } 18\end{array}$ & - & $\begin{array}{l}\mathrm{O}_{2}, \mathrm{PDE} 5 \mathrm{i} \\
\text { treprostinil }\end{array}$ & $\begin{array}{c}29 \text { years: death } \\
\text { from refractory } \\
\text { PH }\end{array}$ \\
\hline 5 & Female & & 40 & $\begin{array}{c}\text { PPHN } \\
\text { Pneumothorax }\end{array}$ & $\begin{array}{c}\text { Yes } \\
10\end{array}$ & Yes & Yes & $\begin{array}{l}\text { Yes } \\
45\end{array}$ & No & $E$ & $\begin{array}{l}2.5 \text { months: } \\
\text { hypoxaemia }\end{array}$ & - & $\begin{array}{c}\mathrm{O}_{2}, \mathrm{PDE} 5 \mathrm{i}, \\
\text { treprostinil, } \\
\text { epoprostenol, CCB }\end{array}$ & $\begin{array}{c}15 \text { years: stable } \\
\mathrm{PH}\end{array}$ \\
\hline 6 & Female & Caucasian & $\begin{array}{l}391 / 7 \\
3.136 \\
-0.27\end{array}$ & RDS & No & No & No & $\begin{array}{l}\text { Yes } \\
12\end{array}$ & $\mathrm{O}_{2}$ & $\mathrm{C}$ & $\begin{array}{l}6 \text { months: } \\
\text { hypoxaemia, } \\
\text { dyspnoea }\end{array}$ & - & ERA, PDE5i, $\mathrm{O}_{2}$ & $\begin{array}{c}11 \text { years: severe } \\
\mathrm{PH}\end{array}$ \\
\hline 7 & Female & Caucasian & $\begin{array}{c}374 / 7 \\
1890 \\
-2.34\end{array}$ & PPHN & No & $\begin{array}{l}\text { Yes } \\
1\end{array}$ & $\begin{array}{l}\text { Yes } \\
13\end{array}$ & $\begin{array}{l}\text { Yes } \\
180\end{array}$ & $\mathrm{O}_{2}$ & $E$ & $\begin{array}{l}1.5 \text { months: } \\
\text { hypoxaemia, } \\
\text { dyspnoea }\end{array}$ & $\begin{array}{l}3 \text { month } \\
\text { biopsy }\end{array}$ & $\begin{array}{l}\mathrm{O}_{2}, \text { iNO, PDE5i, } \\
\text { digoxin }\end{array}$ & $\begin{array}{l}8 \text { months: death, } \\
\text { PH crisis during } \\
\text { surgery (Meckel's } \\
\text { diverticulectomy) }\end{array}$ \\
\hline 8 & Female & Caucasian & 40 & Normal & No & No & No & Yes & No & C & $\begin{array}{l}3 \text { years: } \\
\text { dyspnoea, } \\
\text { fatigability, } \\
\text { syncope }\end{array}$ & - & $\begin{array}{c}\mathrm{O}_{2}, \text { s.c. } \\
\text { treprostinil, PGE5i }\end{array}$ & $\begin{array}{c}12 \text { years: severe } \\
\mathrm{PH}\end{array}$ \\
\hline 9 & Female & Caucasian & $40^{+}$ & $\begin{array}{l}\text { PPHN } \\
\text { MAS }\end{array}$ & $\begin{array}{l}\text { Yes } \\
5\end{array}$ & Yes & $\begin{array}{l}\text { Yes } \\
15\end{array}$ & $\begin{array}{l}\text { Yes } \\
30\end{array}$ & No & C & $\begin{array}{l}2.5 \text { years: } \\
\text { hypoxaemia, } \\
\text { syncope }\end{array}$ & - & $\begin{array}{l}\mathrm{O}_{2}, \text { PDE5i, ERA, } \\
\text { s.c. treprostinil }\end{array}$ & 7 years: severe $\mathrm{PH}$ \\
\hline 10 & Male & Caucasian & $\begin{array}{c}40 \\
3490 \\
-0.17\end{array}$ & PPHN & $\begin{array}{c}\text { Yes } \\
2\end{array}$ & $\begin{array}{l}\text { Yes } \\
2\end{array}$ & $\begin{array}{l}\text { Yes } \\
5\end{array}$ & $\begin{array}{l}\text { Yes } \\
30\end{array}$ & $\mathrm{O}_{2}$ & $\mathrm{C}$ & $\begin{array}{l}18 \text { months: } \\
\text { hypoxaemia }\end{array}$ & $\begin{array}{l}2 \text { years: } \\
\text { biopsy } \\
18 \text { years: } \\
\text { explant }\end{array}$ & $\begin{array}{c}\mathrm{O}_{2} \text {, ERA, PDE5i, } \\
\text { CCB }\end{array}$ & $\begin{array}{l}18 \text { years: } \\
\text { end-stage } \mathrm{PH} \text {, } \\
\text { heart-lung } \\
\text { transplantation }\end{array}$ \\
\hline 11 & Female & & 40 & Normal & No & No & No & Yes & No & $C$ & $\begin{array}{l}8 \text { years: PDA } \\
\text { right to left }\end{array}$ & - & ERA & \\
\hline
\end{tabular}




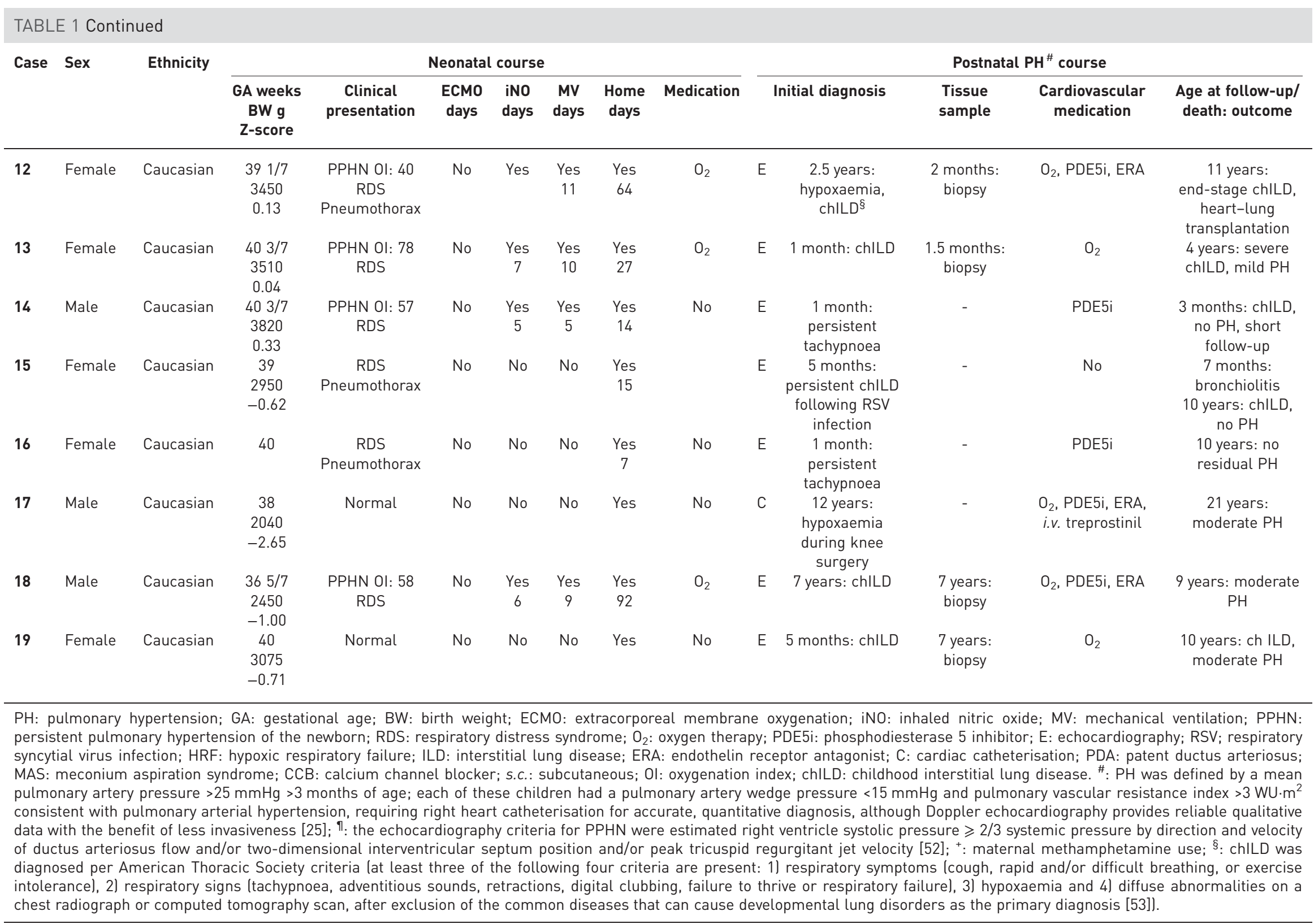




\section{Results}

Genotype characterisation

18 different heterozygous variants were identified in the 19 patients (including two siblings) which consisted of six CNVs involving the 17q23.2 locus and 12 TBX4 SNVs (table 2). The CNVs comprised two sizes of $\sim 2.2 \mathrm{Mb}$ and $\sim 3.6-3.7 \mathrm{Mb}$ encompassing the whole TBX4 coding sequence plus several other genes (figure 1a). The $2.2 \mathrm{Mb} \mathrm{CNV}$ (cases 1, 3, 4 and 5) is a recurrent 17q23.1q23.2 deletion due to segmental duplications that has been previously described [23] and reported in ClinGen. The larger CNV (cases 2 and 6) has only been reported once in ClinVar. The SNVs are novel. Among these, 10 are likely-gene-disrupting variants, including frameshift indel (cases 7-11), premature stop-gain (cases 12-14) and canonical splice site (cases 15 and 16) variants, and three are missense variants affecting the T-box DNA binding domain consensus (cases 17-19) (figure 1b). The two nonsense and two of the frameshift variants located downstream of the T-box domain, in the absence of experimental data demonstrating their gene disrupting effect, were classified as likely pathogenic. The three missense mutations were considered likely pathogenic on the basis of the PolyPhen-2 and/or CADD scores of $0.85-1$ and $\geqslant 10-20$ respectively, conservation of the amino acid position across all vertebrate species, and complete (for $\mathrm{p}$. Gly106 and p.Leu186) or moderate (for p.Val218) conservation in the T-box domain of the 13 human TBX proteins. Of the eight variants in which inheritance was determinable, three (37\%) were de novo and five (62\%) were familial, with carrier siblings affected with SPS (case 18) or determined to have previously had PAH (cases 13 and 14), and carrier mothers affected with SPS (cases 12 and 15), PAH (case 15) or asymptomatic (case 16). Although the number and nature of tested genes varied from centre to centre (supplementary table S1), no BMPR2, forkhead box F1 (FOXF1) or other PH-related pathogenic gene variants could be found in any tested patient.

\section{Clinical phenotype and outcomes}

All patients were born term or late preterm (median 40.0 weeks, interquartile range (IQR) 38.0 weeks), with a female to male ratio of 2.16:1, similar for CNVs and SNVs (tables 1 and 3). Median birthweight was normal for gestational age (median $3075 \mathrm{~g}$, IQR $2450 \mathrm{~g}$ ), although three neonates were small for gestational age (birthweight $\mathrm{z}$-score<-1.28) [24]. 11 neonates required invasive respiratory support. The most frequent presentation was PPHN in 10 out of 19 neonates (53\%), which was severe in eight cases (oxygenation index $>25$ or need for extracorporeal membrane oxygenation (ECMO)). Four neonates $(10 \%)$ presented with transient respiratory distress without $\mathrm{PH}$; the remaining five had an uneventful neonatal course. All patients survived their newborn intensive care unit course and were discharged home at a median age of 37 days (range 7-180 days), six with home oxygen and two with sildenafil. In two patients with PPHN history, right ventricular systolic pressures (RVSP) remained elevated after the neonatal period despite therapy and they both died in infancy, at 5 and 8 months. In the remaining eight with PPHN, $\mathrm{PH}$ appeared to improve or resolve within the first months of life. Later in childhood, the 17 surviving patients underwent a cardiology evaluation, often for new-onset hypoxaemia or for cardiorespiratory symptoms (table 1). These patients were diagnosed with PH (median age 1.5 years, IQR 0.17 years). The duration of follow-up at the time of this report varied between patients, this study being retrospective (median 10.0 years, IQR 4.7 years). Three patients evolved to end-stage lung disease despite multiple vasodilator therapies: one died at 29 years old and two underwent heart-lung transplantation, one at 11 years and one at 18 years old. 11 patients continue to have chronic $\mathrm{PH}$ at their last evaluation, despite the use of multiple $\mathrm{PH}$-targeted therapies in seven patients, a single $\mathrm{PH}$-targeted therapy in one patient, and treatment with supplemental oxygen alone in three patients. $\mathrm{PH}$ had resolved at the last follow-up in the remaining three patients (cases 14, 15 and 16: 3 months-10 years), one of whom was medication-free whereas two remained on single vasodilator therapy. 10 out of the 13 patients whose information was retrievable (77\%) had skeletal anomalies, including SPS with its typical foot anomaly [9]. Other neurological and developmental disorders included autism, microcephaly, neurosensory deficits and muscular tone anomalies.

\section{Cardiac imaging and haemodynamic analysis}

Seven of the patients were assessed by echocardiography alone, while 12 underwent at least one RHC during their treatment course (table 4). 10 patients (53\%) had systemic or suprasystemic RVSP in the neonatal period. Eight had patent ductus arteriosus (PDA), which persisted beyond the neonatal period in four patients. Two of these had left-to-right shunting at initial PH diagnosis: one was surgically closed at age 4 years (case 4), and one was haemodynamically insignificant (case 5). Two patients (cases 5 and 11) had right-to-left shunting at the diagnostic RHC persisting at follow-up. An atrial septal defect was present in eight patients (42\%). In the 12 patients in whom cardiac catheterisation was performed (median age 1.5 years, IQR 0.17 years), high mean pulmonary artery pressures (mPAP) $(60.0 \mathrm{mmHg}$, IQR $57.5 \mathrm{~mm} \mathrm{Hg})$ and pulmonary vascular resistance indices (median 16.6 Wood units, IQR 10.7 Wood units) were 


\begin{tabular}{|c|c|c|c|c|c|c|c|c|c|}
\hline Case & $\begin{array}{l}\text { TBX4 nucleotide and } \\
\text { protein variant }\end{array}$ & $\begin{array}{c}\text { Genomic } \\
\text { position } \\
\text { (Hg19; } \\
\text { Chr17) }^{\#}\end{array}$ & $\begin{array}{c}\text { Putative } \\
\text { mechanism and } \\
\text { consequence }\end{array}$ & $\begin{array}{l}\text { Genotype and } \\
\text { inheritance }\end{array}$ & $\begin{array}{l}\text { ExAC allele } \\
\text { frequency, } \\
\text { CADD score }\end{array}$ & $\begin{array}{c}\text { ACMG } \\
\text { classification }\end{array}$ & $\begin{array}{c}\text { Skeletal } \\
\text { anomalies }\end{array}$ & Other anomalies & $\begin{array}{l}\text { Neurological and } \\
\text { psychomotor } \\
\text { deficits }\end{array}$ \\
\hline 1 & CNV & $\begin{array}{c}17 q 23.1 q 23.2 \\
(58078171-60 \\
316749) \times 1\end{array}$ & $\begin{array}{c}\text { Gene deletion } \\
\text { haploinsufficiency }\end{array}$ & $\begin{array}{l}\text { Heterozygous } \\
\text { De novo }\end{array}$ & $\mathrm{n} / \mathrm{a}$ & $\begin{array}{l}\text { Pathogenic } \\
\text { (ClinGen) }\end{array}$ & Not known & $\begin{array}{l}\text { Hypothyroidism, } \\
\text { cortisol deficiency }\end{array}$ & Hypertonia \\
\hline 2 & CNV & $\begin{array}{c}17 q 22 q 23.2(56 \\
623275-60 \\
285107) \times 1\end{array}$ & $\begin{array}{c}\text { Gene deletion } \\
\text { haploinsufficiency }\end{array}$ & $\begin{array}{l}\text { Heterozygous } \\
\text { Not known }\end{array}$ & $\mathrm{n} / \mathrm{a}$ & $\begin{array}{l}\text { Likely } \\
\text { pathogenic } \\
\text { (ClinVar) }\end{array}$ & $\begin{array}{c}\text { Facial } \\
\text { dysmorphism, } \\
\text { foot anomaly, } \\
\text { not known }\end{array}$ & ASD, omphalocele & $\begin{array}{l}\text { Developmental } \\
\text { delay, seizures, } \\
\text { nystagmus }\end{array}$ \\
\hline 3 & CNV & $\begin{array}{c}17 q 23.1 q 23.2 \\
(57972342-60 \\
472864) \times 1\end{array}$ & $\begin{array}{c}\text { Gene deletion } \\
\text { haploinsufficiency }\end{array}$ & $\begin{array}{l}\text { Heterozygous } \\
\text { Not known }\end{array}$ & $\mathrm{n} / \mathrm{a}$ & $\begin{array}{l}\text { Likely } \\
\text { pathogenic } \\
\text { (ClinGen) }\end{array}$ & $\begin{array}{l}\text { Joint } \\
\text { contractures, } \\
\text { foot anomaly }\end{array}$ & ASD & $\begin{array}{c}\text { Mild } \\
\text { developmental } \\
\text { delay, hearing } \\
\text { loss, two-vessel } \\
\text { cord, } \\
\text { vesicoureteral } \\
\text { reflux }\end{array}$ \\
\hline 4 & CNV & $\begin{array}{l}17 q 23.1(58 \\
313766-60 \\
315220) \times 1\end{array}$ & $\begin{array}{c}\text { Gene deletion } \\
\text { haploinsufficiency }\end{array}$ & $\begin{array}{l}\text { Heterozygous } \\
\text { Not known }\end{array}$ & $\mathrm{n} / \mathrm{a}$ & $\begin{array}{l}\text { Likely } \\
\text { pathogenic } \\
\text { (ClinGen) }\end{array}$ & Foot anomaly & $\begin{array}{l}\text { PDA (ligation at } \\
\quad 4 \text { years) }\end{array}$ & $\begin{array}{c}\text { Mild } \\
\text { developmental } \\
\text { delay }\end{array}$ \\
\hline 5 & CNV & $\begin{array}{c}17 q 23.1 q 23.2 \\
(57992012-60 \\
330998) \times 1\end{array}$ & $\begin{array}{l}\text { Gene deletion } \\
\text { haploinsufficiency }\end{array}$ & $\begin{array}{l}\text { Heterozygous } \\
\text { Not known }\end{array}$ & $\mathrm{n} / \mathrm{a}$ & $\begin{array}{l}\text { Likely } \\
\text { pathogenic } \\
\text { (ClinGen) }\end{array}$ & $\begin{array}{l}\text { SPS, foot } \\
\text { anomaly }\end{array}$ & PDA, ASD, VSD & $\begin{array}{l}\text { Developmental } \\
\text { delay, nystagmus }\end{array}$ \\
\hline 6 & CNV & $\begin{array}{c}17 q 22 q 23.2(56 \\
429075-60 \\
181763) \times 1\end{array}$ & $\begin{array}{c}\text { Gene deletion } \\
\text { haploinsufficiency }\end{array}$ & $\begin{array}{l}\text { Heterozygous } \\
\text { Not known }\end{array}$ & $\mathrm{n} / \mathrm{a}$ & $\begin{array}{l}\text { Likely } \\
\text { pathogenic }\end{array}$ & Club foot & ASD, gastrostomy & $\begin{array}{l}\text { Microcephaly, } \\
\text { hearing loss, } \\
\text { esotropia, } \\
\text { nystagmus, } \\
\text { severe } \\
\text { developmental } \\
\text { delay }\end{array}$ \\
\hline 7 & c.251_delG p.(Gly84Alafs*4) & 59534962 & $\begin{array}{c}\text { Indel } \\
\text { Frameshift } \\
\text { Loss of function }\end{array}$ & $\begin{array}{l}\text { Heterozygous } \\
\text { De novo }\end{array}$ & ExAC AF: 0 & Pathogenic & Not known & $\begin{array}{c}\text { Transient PDA, } \\
\text { failure to thrive, } \\
\text { Meckel } \\
\text { diverticulum }\end{array}$ & $\begin{array}{l}\text { Developmental } \\
\text { delay }\end{array}$ \\
\hline 8 & $\begin{array}{c}\text { c.847dupC } p . \\
\text { (Gln283Profs*103) }\end{array}$ & 59557506 & $\begin{array}{c}\text { Indel } \\
\text { Frameshift } \\
\text { Loss of function }\end{array}$ & $\begin{array}{l}\text { Heterozygous } \\
\text { Not known }\end{array}$ & ExAC AF: 0 & $\begin{array}{l}\text { Likely } \\
\text { pathogenic }\end{array}$ & Not known & & No \\
\hline 9 & c.146delG p.(Gly49Aspfs*39) & 59533997 & $\begin{array}{c}\text { Indel } \\
\text { Frameshift } \\
\text { Loss of function }\end{array}$ & $\begin{array}{l}\text { Heterozygous } \\
\text { Not known }\end{array}$ & ExAC AF: 0 & $\begin{array}{l}\text { Likely } \\
\text { pathogenic }\end{array}$ & Not known & $\begin{array}{l}\text { ASD, obstructive } \\
\text { apnoea }\end{array}$ & No \\
\hline 10 & $\begin{array}{c}\text { c.538_547delCCCTTTGGCC } \\
\text { p.(Pro180llefs*45) }\end{array}$ & $\begin{array}{c}59545007-59 \\
545016\end{array}$ & $\begin{array}{c}\text { Indel } \\
\text { Frameshift } \\
\text { Loss of function }\end{array}$ & $\begin{array}{l}\text { Heterozygous } \\
\text { Not known }\end{array}$ & ExAC AF: 0 & $\begin{array}{l}\text { Likely } \\
\text { pathogenic } \\
\text { (ClinVar) }\end{array}$ & $\begin{array}{l}\text { SPS, foot } \\
\text { anomaly }\end{array}$ & & No \\
\hline 11 & $\begin{array}{l}\text { c.1112-1113insC p. } \\
\text { (Pro371Profs*15) }\end{array}$ & $\begin{array}{c}59560348-59 \\
560349\end{array}$ & $\begin{array}{c}\text { Indel } \\
\text { Frameshift } \\
\text { Loss of function }\end{array}$ & $\begin{array}{l}\text { Heterozygous } \\
\text { Not known }\end{array}$ & ExAC AF: 0 & $\begin{array}{l}\text { Likely } \\
\text { pathogenic } \\
\text { (ClinVar) }\end{array}$ & Foot anomaly & PDA & No \\
\hline
\end{tabular}




\begin{tabular}{|c|c|c|c|c|c|c|c|c|c|}
\hline Case & $\begin{array}{l}\text { TBX4 nucleotide and } \\
\text { protein variant }\end{array}$ & $\begin{array}{l}\text { Genomic } \\
\text { position } \\
\text { (Hg19; } \\
\text { Chr17) }\end{array}$ & $\begin{array}{c}\text { Putative } \\
\text { mechanism and } \\
\text { consequence }\end{array}$ & $\begin{array}{l}\text { Genotype and } \\
\text { inheritance }\end{array}$ & $\begin{array}{l}\text { ExAC allele } \\
\text { frequency, } \\
\text { CADD score }\end{array}$ & $\begin{array}{c}\text { ACMG } \\
\text { classification }\end{array}$ & $\begin{array}{l}\text { Skeletal } \\
\text { anomalies }\end{array}$ & Other anomalies & $\begin{array}{l}\text { Neurological and } \\
\text { psychomotor } \\
\text { deficits }\end{array}$ \\
\hline 12 & c. $1054 \mathrm{C}>\mathrm{T}$ p. $(\operatorname{Arg} 352 *)$ & 59560290 & $\begin{array}{l}\text { Nonsense AA } \\
\text { substitution } \\
\text { Loss of function }\end{array}$ & $\begin{array}{l}\text { Heterozygous } \\
\text { Heritable: } \\
\text { (M) c.1054C>T } \\
\text { with SPS }\end{array}$ & ExAC AF: 0 & $\begin{array}{l}\text { Likely } \\
\text { pathogenic }\end{array}$ & $\begin{array}{l}\text { SPS, pelvis and } \\
\text { foot anomaly }\end{array}$ & ASD, ILD & No \\
\hline $13^{\pi}$ & c. $1018 \mathrm{C}>\mathrm{T}$ p. $\left(\operatorname{Arg} 340^{*}\right)$ & 59557677 & $\begin{array}{l}\text { Nonsense AA } \\
\text { substitution } \\
\text { Loss of function }\end{array}$ & $\begin{array}{l}\text { Heterozygous } \\
\text { Heritable: both } \\
\text { siblings } \\
\text { carrying p. } \\
\text { (Arg340*); } \\
\text { parents not } \\
\text { tested }\end{array}$ & ExAC AF: 0 & $\begin{array}{l}\text { Likely } \\
\text { pathogenic }\end{array}$ & No & $\begin{array}{c}\text { Transient PDA and } \\
\text { PFO, ILD }\end{array}$ & No \\
\hline $14^{9}$ & c.1018C>T p.(Arg340*) & 59557677 & $\begin{array}{l}\text { Nonsense AA } \\
\text { substitution } \\
\text { Loss of function }\end{array}$ & $\begin{array}{l}\text { Heterozygous } \\
\text { Heritable: both } \\
\text { siblings } \\
\text { carrying p. } \\
\text { (Arg340*); } \\
\text { parents not } \\
\text { tested }\end{array}$ & ExAC AF: 0 & $\begin{array}{l}\text { Likely } \\
\text { pathogenic }\end{array}$ & No & $\begin{array}{c}\text { Transient PFO and } \\
\text { PDA, ILD }\end{array}$ & Unknown \\
\hline 15 & c.792-1G>C & 59557450 & $\begin{array}{l}\text { Splice site AA } \\
\text { substitution } \\
\text { Loss of function }\end{array}$ & $\begin{array}{c}\text { Heterozygous } \\
\text { Heritable: (M) } \\
\text { c.792-1G>C } \\
\text { with SPS, PH, } \\
\text { ILD }\end{array}$ & ExAC AF: 0 & $\begin{array}{l}\text { Likely } \\
\text { pathogenic }\end{array}$ & Foot anomaly & $\begin{array}{l}\text { Mandibular } \\
\text { angioma }\end{array}$ & No \\
\hline 16 & c. $702+1 G>A$ & 59556141 & $\begin{array}{l}\text { Splice site AA } \\
\text { substitution } \\
\text { Loss of function }\end{array}$ & $\begin{array}{c}\text { Heterozygous } \\
\text { Heritable: }(\mathrm{M}) \\
\text { c. } 702+1 \mathrm{G}>\mathrm{A}\end{array}$ & ExAC AF: 0 & $\begin{array}{l}\text { Likely } \\
\text { pathogenic } \\
\text { (ClinVar) }\end{array}$ & Not known & No & No \\
\hline 17 & c.316G>A p.(Gly106Ser) & 59534214 & $\begin{array}{l}\text { Missense AA } \\
\text { substitution }\end{array}$ & $\begin{array}{l}\text { Heterozygous } \\
\text { De novo }\end{array}$ & $\begin{array}{c}\text { ExAC AF: } \\
0.000008236 \\
\text { CADD: } 11.0 \\
\text { P2: } 1.000\end{array}$ & $\begin{array}{l}\text { Likely } \\
\text { pathogenic }\end{array}$ & $\begin{array}{l}\text { SPS, pelvis and } \\
\text { foot scoliosis }\end{array}$ & $\begin{array}{c}\text { PDA, short stature, } \\
\text { angiofibromas, } \\
\text { keratoconus }\end{array}$ & ADHD, autism \\
\hline 18 & c.557T>G p.(Leu186Arg) & 59555995 & $\begin{array}{l}\text { Missense AA } \\
\text { substitution }\end{array}$ & $\begin{array}{l}\text { Heterozygous } \\
\text { Heritable: two } \\
\text { siblings } \\
\text { carrying p. } \\
\text { (Leu186Arg), } \\
\text { with SPS }\end{array}$ & $\begin{array}{l}\text { ExAC AF: } 0 \\
\text { CADD: } 28.8 \\
\text { P2: } 1.000\end{array}$ & $\begin{array}{l}\text { Likely } \\
\text { pathogenic } \\
\text { (ClinVar) }\end{array}$ & $\begin{array}{l}\text { Pelvis, vertebral } \\
\text { and foot } \\
\text { anomaly }\end{array}$ & $\begin{array}{c}\text { ASD, PDA, short } \\
\text { stature, facial } \\
\text { dysmorphism (long } \\
\text { philtrum, } \\
\text { hypertelorism) }\end{array}$ & $\begin{array}{c}\text { Moderate } \\
\text { developmental } \\
\text { delay, hypotonia }\end{array}$ \\
\hline 19 & c.652G>A p.(Val218Met) & 59556090 & $\begin{array}{l}\text { Missense AA } \\
\text { substitution }\end{array}$ & $\begin{array}{l}\text { Heterozygous } \\
\text { Not tested }\end{array}$ & $\begin{array}{l}\text { ExAC AF: } \\
0.0001153 \\
\text { CADD: } 24.2 \\
\text { P2: } 0.635\end{array}$ & $\begin{array}{l}\text { Likely } \\
\text { pathogenic }\end{array}$ & No & $\begin{array}{l}\text { PFO, short stature, } \\
\text { hearing loss }\end{array}$ & Microcephaly \\
\hline
\end{tabular}

TBX4: T-box transcription factor 4; Hg19: Homo sapiens genome assembly GRCh37; Chr17: chromosome 17; ExAC: Exome Aggregation Consortium; CADD: Combined Annotation Dependent Depletion; ACMG: American College of Medical Genetics; CNV: copy number variant; n/a: not available; ASD: atrial septal defect; PDA: patent ductus arteriosus; SPS: small patella syndrome; VSD: ventricular septal defect; AF: allele frequency; AA: amino acid; M: mother; ILD: interstitial lung disease; PFO: patent foramen ovale; PH: pulmonary hypertension; P2: PolyPhen2; ADHD: attention deficit hyperactivity disorder. \#: genomic annotations were based on NC_000017.11: homo sapiens chromosome 17, GRCh38.p7 primary assembly; transcript sequence NM_001321120.1; protein sequence NP_001308049.1; ${ }^{\text {ๆ: }}$ siblings. 

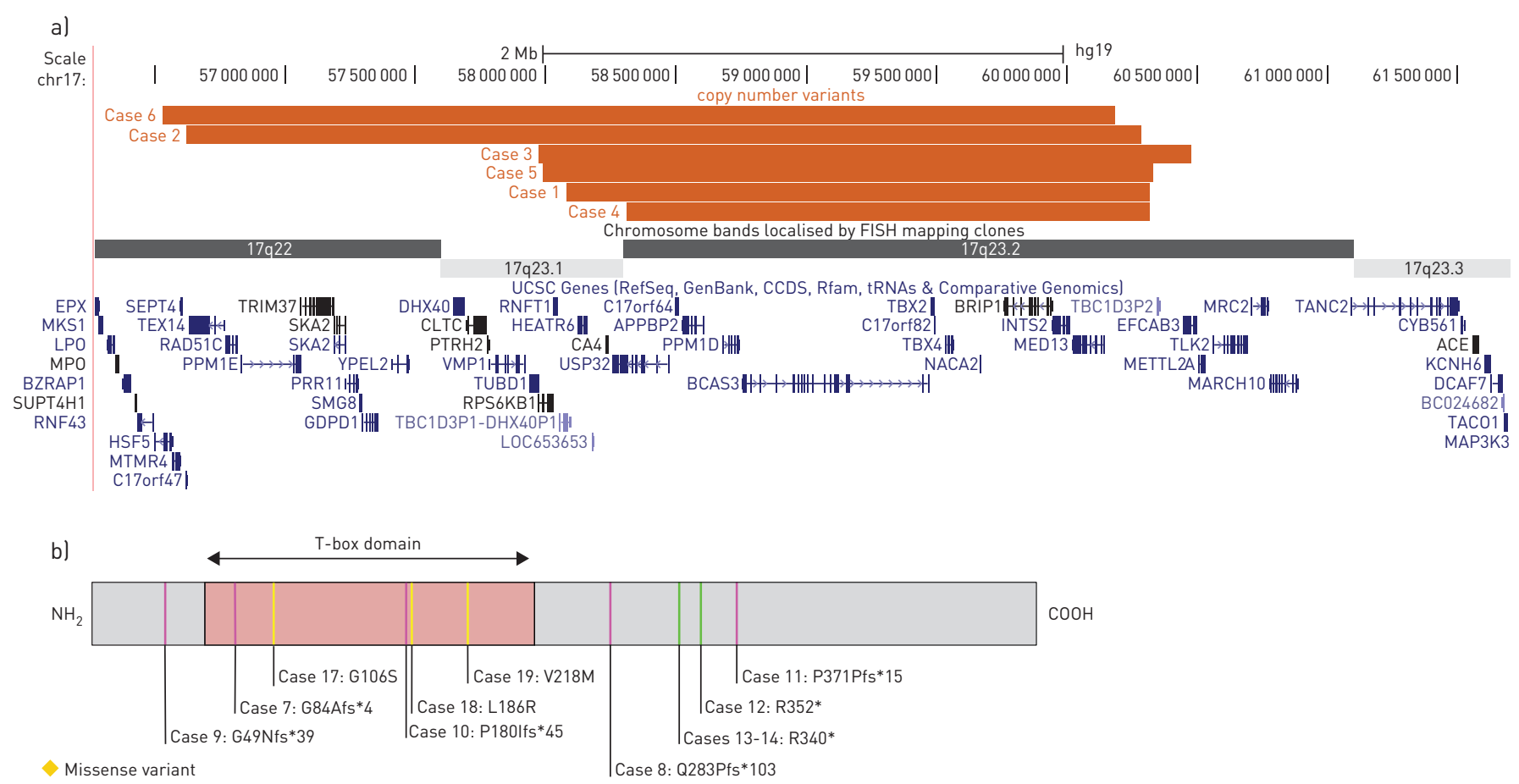

Nonsense variant

Frameshift variant

FIGURE 1 Position of 17q23 deletions and TBX4 mutations. a) Genomic deletions identified in the 17q23.2 region. The smallest deletion overlap region encompasses the following coding genes: MED13 (subunit of the large Mediator complex, ubiquitous), INTS2 (subunit of the Integrator complex, low lung expression), BRIP1 (a member of the DEAH helicase family contributing to BRCA1 activity, ubiquitous), BCAS3 (associated with angiogenesis and related processes such as cell adhesion, extracellular matrix organisation, peptidase activity and transforming growth factor- $\beta$ signalling, ubiquitous with high expression in lung [49]), TBX4 (transcription factor involved in several developmental processes including hindlimb, lung and allantois), TBX2 (transcription factor involved in patterning of several organs, including heart, brain and limbs [42]), PPM1D ( protein phosphatase that regulates the DNA damage response pathway, mostly expressed in brain and associated with developmental disability in human [50], APPBP2 (ubiquitous, interacts with microtubules and is associated with transport and/or processing) and USP32 (an ubiquitin-specific protease). Among these genes, only TBX4, TBX2 and PPM1D have rare variants described in human disease. University of California Santa Cruz (UCSC) Genome Browser (GRCH37/Hg19) [51]. b) Mutation positions in TBX4 protein. The TBX4 protein is represented in grey, with the T-box domain in pink. The three missense variants (yellow) are located in the T-box domain. Three of the frameshift variants (purple) are located either upstream or inside the T-box domain, while two are located downstream. The two nonsense mutations (green) are located downstream. FISH: fluorescent in situ hybridisation; CCDS: Consensus Coding Sequence project; tRNA: transfer RNA.

demonstrated. However, only six out of 12 patients met all criteria for a strict diagnosis of PAH based on American Thoracic Society guidelines [25]. In the six patients with serial RHCs, mPAP and pulmonary vascular resistance indices values were equally elevated or increased at follow-up (data not shown). Among the 10 patients who underwent acute vasoreactivity testing, eight (80\%) failed to show a decrease in mPAP of at least $10 \mathrm{mmHg}$ to $<40 \mathrm{mmHg}$ [25]. Six had a reduced pulmonary-to-systemic blood flow ratio, indicating significant right-to-left shunts. When performed, pulmonary angiography revealed diffuse anatomical and vascular anomalies, including tortuous pulmonary arterioles, abnormal capillary blush, small pulmonary veins and venules, and pulmonary venous obstruction in one patient (not shown).

\section{Phenotypic characteristics and variant type}

Table 3 compares the clinical, functional features between the six CNV and the 13 SNV carriers. Although we observed a greater prevalence of associated cardiac and foot anomalies in CNV carriers, only developmental disability reached statistical significance ( $100 \%$ versus $33 \%, \mathrm{p}=0.029)$, in line with others' findings [10]. We also observed a trend for greater RHC functional severity in SNV carriers, although this may reflect an older age at first catheterisation in that group. Supplementary table S3, which compares published 17q23 deletions inclusive and exclusive of the TBX2/TBX4 loci including our series, shows a greater prevalence of congenital heart defects $(57 \%$ versus $0 \%, \mathrm{p}=0.02)$ and a similar trend for the presence of $\mathrm{PH}(57 \%$ versus $17 \%, \mathrm{p}=0.16)$ for TBX2/TBX4-inclusive deletions.

\section{Imaging studies}

Thoracic images could be only collected in a subset of cases (figure 2, supplementary table S2). Neonatal chest radiography $(n=5)$ showed lung hypoplasia (figure $2 \mathrm{a}$ ), air leaks and/or ground-glass opacities; chest 
TABLE 3 Clinical and functional differences in patients with chromosomal deletions versus SNVs

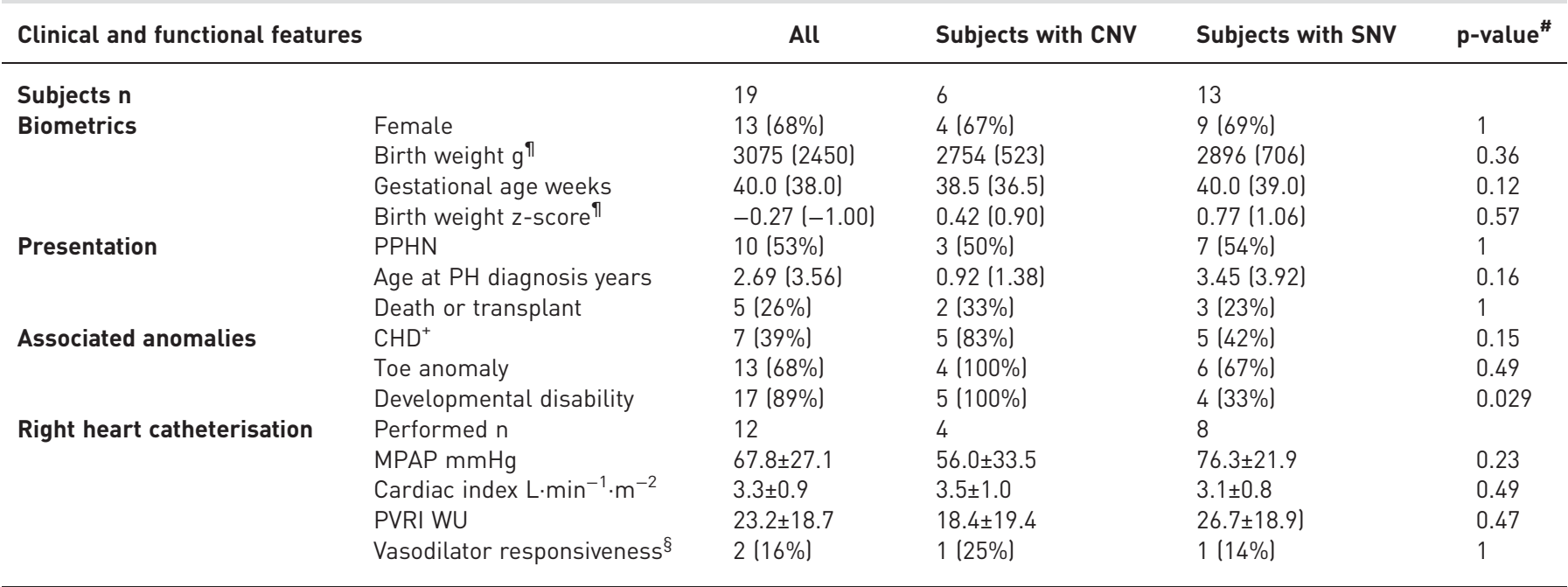

Data are presented as $\mathrm{n}(\%)$, median (interquartile range) or mean \pm SD, unless otherwise stated. CNV: copy number variant; SNV: small nucleotide variant; PPHN: persistent pulmonary hypertension of the newborn; PH: pulmonary hypertension; CHD: congenital heart defect; MPAP: mean pulmonary arterial pressure; PVRI: pulmonary vascular resistance index; WU: Wood units. \#: p-value for comparison of the group with CNVs versus SNVs; t-test for numerical values, Fisher test for categorical values; ${ }^{\text {ๆ: }}$ birth weight only available for 13 patients; ${ }^{+}$: $\mathrm{CHD}$ was not determined in one patient due the presence of patent ductus arteriosus and patent foramen ovale at $<1$ month of age, not re-tested subsequently; ${ }^{\S}$ : vasodilator responsiveness categorised according to Revised Barst Criteria [31].

radiography in infancy and early childhood $(n=4)$ showed a pattern of septal thickening with multifocal areas of dysventilation, bronchial thickening and ground-glass opacities (figure 2b). Computed tomography (CT) scans obtained between 1 and 18 years of age $(n=5)$ showed a spectrum of findings, including multifocal ground-glass opacities, honeycombing and alternating focal cystic changes, and condensed areas and nodules suggesting lobular and lobar fibrosis (figure 2c-f).

\section{Lung histopathology}

Pathologic material was available for seven patients, and histological features of lung development and vessel remodelling were analysed semi-quantitatively (table 5). All samples showed diffuse alveolar growth abnormality and variable degrees of pulmonary artery wall remodelling with or without fibrointimal proliferation. No plexiform lesions or vessel necrosis were noted. In patients who had severe symptoms at an early age and underwent biopsy in the neonatal period (cases 1, 7, 12 and 13; figure 3a-i), the histology showed severe disruption of distal lung development characterised by delayed lobular growth with dilated distal airspaces and immature-appearing alveoli without secondary septa, often lined by reactive cuboidal epithelial cells. The distal airspaces appeared enlarged with simplified alveoli. In all cases, there were signs of thickened interstitium; three showed the presence of pale and immature mesenchymal cells as observed in pulmonary interstitial glycogenosis [26], and three had patchy interstitial fibrosis. All had evidence of pulmonary arterial hypertensive remodelling. Back-to-back bronchiolar profiles were seen in two cases and one showed the presence of bronchial vessel recruitment, including intrapulmonary bronchopulmonary anastomoses (IBAs). Overall, these structural changes point to severe disruption of all compartments of distal lung development, reflecting growth arrest during the canalicular or early saccular stage. The lung histology of patients who underwent biopsy in childhood (cases 10a, 18 and 19, and 10b-explant; figure 3j-r) showed evidence of recruited bronchial vascular system, including IBAs and expanded bronchial veins and capillaries, in addition to alveolar simplification and pulmonary artery remodelling. Features of airway remodelling and functional compromise were variably present, characterised by airway wall thickening, increased number of intra-alveolar macrophages and multinucleated giant cells with cholesterol crystals (not shown).

A longitudinal histological analysis was possible in case 10 (biopsy at 2 years and transplant at 18 years). The most striking histological findings included the progression of compromised airway/alveolar growth, characterised by multifocal, markedly underdeveloped and tortuous back-to-back bronchiolar structures, similar to those seen in congenital pulmonary airway malformations [27] or acinar dysplasia (AD) [15]. In addition, pulmonary arteries, lymphatic vessels, airways and pleural vessels showed marked medial wall thickening, and areas of bone formation were noted in the subsequent explant suggesting mesenchymal 


\begin{tabular}{|c|c|c|c|c|c|c|c|c|c|c|c|c|c|c|c|c|c|c|}
\hline \multirow[t]{3}{*}{ Case } & \multirow{2}{*}{\multicolumn{2}{|c|}{$\begin{array}{c}\text { Original } \\
\text { echocardiography }\end{array}$}} & \multirow{3}{*}{$\begin{array}{l}\text { Heart } \\
\text { defects }\end{array}$} & \multicolumn{15}{|c|}{ Original right heart catheterisation data } \\
\hline & & & & \multicolumn{8}{|c|}{ Haemodynamics at baseline } & \multicolumn{7}{|c|}{ Haemodynamics on maximum $\mathrm{O}_{2} /$ iNO vasodilation ${ }^{\#}$} \\
\hline & $\begin{array}{l}\text { Age } \\
\text { years }\end{array}$ & $\begin{array}{c}\text { Estimated } \\
\text { RVSP } \mathrm{mmHg}\end{array}$ & & $\begin{array}{c}\text { Age } \\
\text { years }\end{array}$ & $\begin{array}{l}\text { mPAP } \\
\text { mmHg }\end{array}$ & $\begin{array}{c}\text { MSP } \\
\mathrm{mmHg}\end{array}$ & $\begin{array}{l}\text { PCWP } \\
\mathrm{mmHg}\end{array}$ & PVRi & $\begin{array}{c}\mathrm{RA} \\
\mathrm{mmHg}\end{array}$ & $\frac{\mathrm{Cl}}{\mathrm{L} \cdot \mathrm{min}^{-1} \cdot \mathrm{m}^{-2}}$ & $\begin{array}{l}\text { Qp/ } \\
\text { Qs }\end{array}$ & $\begin{array}{l}\mathrm{mPAP} \\
\mathrm{mmHg}\end{array}$ & $\begin{array}{l}\text { PCWP } \\
\mathrm{mmHg}\end{array}$ & PVRi & $\begin{array}{c}\mathrm{RA} \\
\mathrm{mmHg}\end{array}$ & $\frac{\mathrm{Cl}}{\mathrm{L} \cdot \mathrm{min}^{-1} \cdot \mathrm{m}^{-2}}$ & $\begin{array}{l}\text { Qp/ } \\
\text { Qs }\end{array}$ & Response \\
\hline 1 & 0.3 & Suprasystemic & None & $\mathrm{n} / \mathrm{a}$ & $\mathrm{n} / \mathrm{a}$ & $\mathrm{n} / \mathrm{a}$ & $\mathrm{n} / \mathrm{a}$ & $\mathrm{n} / \mathrm{a}$ & $\mathrm{n} / \mathrm{a}$ & $\mathrm{n} / \mathrm{a}$ & $\mathrm{n} / \mathrm{a}$ & $\mathrm{n} / \mathrm{a}$ & $\mathrm{n} / \mathrm{a}$ & $\mathrm{n} / \mathrm{a}$ & $\mathrm{n} / \mathrm{a}$ & $\mathrm{n} / \mathrm{a}$ & $\mathrm{n} / \mathrm{a}$ & $\mathrm{n} / \mathrm{a}$ \\
\hline 2 & 0.5 & $>2 / 3$ systemic & ASD & 1.0 & 22 & 56 & 7.5 & 4.14 & 6 & 2.9 & 0.91 & 18 & 6 & 5.59 & 6 & 2.4 & 0.89 & $\mathrm{~N}$ \\
\hline 3 & 0.15 & Systemic & ASD & 0.2 & 38 & 48 & 7 & 6.7 & 5 & 4.3 & 0.9 & 30 & 7 & 2.8 & 5 & 6.1 & 1.4 & Y \\
\hline 4 & 4 & $<$ Systemic ${ }^{\pi}$ & $\mathrm{PDA}^{\pi}$ & 18 & 102 & 92 & 24 & 50 & 15 & 2.4 & 0.6 & 75 & $\mathrm{n} / \mathrm{a}$ & 18.6 & $\mathrm{n} / \mathrm{a}$ & 2 & 1.8 & $\mathrm{~N}$ \\
\hline 5 & 0.2 & Suprasystemic & $\begin{array}{l}\text { PDA, } \\
\text { ASD, } \\
\text { VSD }\end{array}$ & 0.3 & 80 & 73 & $\mathrm{n} / \mathrm{a}$ & 24 & 6 & 4.5 & 0.6 & 70 & 10 & 23 & $\mathrm{n} / \mathrm{a}$ & 2.8 & 0.9 & $\mathrm{~N}$ \\
\hline 6 & 0.5 & Systemic & ASD & 0.5 & 38 & $\mathrm{n} / \mathrm{a}$ & $\mathrm{n} / \mathrm{a}$ & 7.0 & 4 & $\mathrm{n} / \mathrm{a}$ & 1.1 & $\mathrm{n} / \mathrm{a}$ & $\mathrm{n} / \mathrm{a}$ & 4.5 & $\mathrm{n} / \mathrm{a}$ & $\mathrm{n} / \mathrm{a}$ & $\mathrm{n} / \mathrm{a}$ & $\mathrm{n} / \mathrm{a}$ \\
\hline 7 & 0.1 & Suprasystemic & None & $\mathrm{n} / \mathrm{a}$ & $\mathrm{n} / \mathrm{a}$ & $\mathrm{n} / \mathrm{a}$ & $\mathrm{n} / \mathrm{a}$ & $\mathrm{n} / \mathrm{a}$ & $\mathrm{n} / \mathrm{a}$ & $\mathrm{n} / \mathrm{a}$ & $\mathrm{n} / \mathrm{a}$ & $\mathrm{n} / \mathrm{a}$ & $\mathrm{n} / \mathrm{a}$ & $\mathrm{n} / \mathrm{a}$ & $\mathrm{n} / \mathrm{a}$ & $\mathrm{n} / \mathrm{a}$ & $\mathrm{n} / \mathrm{a}$ & $\mathrm{n} / \mathrm{a}$ \\
\hline 8 & 4.4 & Suprasystemic & None & 4.4 & 83 & 60 & 11 & 27.4 & 6 & 2.6 & 1 & 78 & 9 & 23.4 & 7 & 2.9 & 1 & $\mathrm{~N}$ \\
\hline 9 & 2.5 & Suprasystemic & ASD & 2.4 & 99 & 58 & 16 & 65.1 & 9 & 1.8 & 0.8 & $\mathrm{n} / \mathrm{a}$ & $\mathrm{n} / \mathrm{a}$ & 27.9 & $\mathrm{n} / \mathrm{a}$ & 4.6 & 0.5 & $\mathrm{~N}$ \\
\hline 10 & 1.5 & $>2 / 3$ systemic & None & 1.5 & 66 & 70 & 10 & 16.5 & 6 & 4.0 & 1.0 & 23 & $\mathrm{n} / \mathrm{a}$ & 3.2 & $\mathrm{n} / \mathrm{a}$ & $\mathrm{n} / \mathrm{a}$ & 1.0 & Y \\
\hline 11 & 7.7 & Suprasystemic & PDA & 7.7 & 63 & 60 & 8 & 18.3 & 5 & 3.8 & 0.8 & 63 & 8 & 19.6 & 6 & 3.1 & 0.9 & $\mathrm{~N}$ \\
\hline 12 & 2.5 & Suprasystemic & None & 7.0 & 111 & 92 & $\mathrm{n} / \mathrm{a}$ & 36.1 & 2 & 2.4 & 1.12 & 110 & 102 & 35.5 & $\mathrm{n} / \mathrm{a}$ & 2.6 & 0.9 & $\mathrm{~N}$ \\
\hline 13 & 0.1 & $>2 / 3$ systemic & ASD & $\mathrm{n} / \mathrm{a}$ & $\mathrm{n} / \mathrm{a}$ & $\mathrm{n} / \mathrm{a}$ & $\mathrm{n} / \mathrm{a}$ & $\mathrm{n} / \mathrm{a}$ & $\mathrm{n} / \mathrm{a}$ & $\mathrm{n} / \mathrm{a}$ & $\mathrm{n} / \mathrm{a}$ & $\mathrm{n} / \mathrm{a}$ & $\mathrm{n} / \mathrm{a}$ & $\mathrm{n} / \mathrm{a}$ & $\mathrm{n} / \mathrm{a}$ & $\mathrm{n} / \mathrm{a}$ & $\mathrm{n} / \mathrm{a}$ & $\mathrm{n} / \mathrm{a}$ \\
\hline 14 & 0.1 & $>2 / 3$ systemic & $\mathrm{n} / \mathrm{a}$ & $\mathrm{n} / \mathrm{a}$ & $\mathrm{n} / \mathrm{a}$ & $\mathrm{n} / \mathrm{a}$ & $\mathrm{n} / \mathrm{a}$ & $\mathrm{n} / \mathrm{a}$ & $\mathrm{n} / \mathrm{a}$ & $\mathrm{n} / \mathrm{a}$ & $\mathrm{n} / \mathrm{a}$ & $\mathrm{n} / \mathrm{a}$ & $\mathrm{n} / \mathrm{a}$ & $\mathrm{n} / \mathrm{a}$ & $\mathrm{n} / \mathrm{a}$ & $\mathrm{n} / \mathrm{a}$ & $\mathrm{n} / \mathrm{a}$ & $\mathrm{n} / \mathrm{a}$ \\
\hline 15 & 5 & $\begin{array}{c}>2 / 3 \text { systemic } \\
\text { (30) }\end{array}$ & None & $\mathrm{n} / \mathrm{a}$ & $\mathrm{n} / \mathrm{a}$ & $\mathrm{n} / \mathrm{a}$ & $\mathrm{n} / \mathrm{a}$ & $\mathrm{n} / \mathrm{a}$ & $\mathrm{n} / \mathrm{a}$ & $\mathrm{n} / \mathrm{a}$ & $\mathrm{n} / \mathrm{a}$ & $\mathrm{n} / \mathrm{a}$ & $\mathrm{n} / \mathrm{a}$ & $\mathrm{n} / \mathrm{a}$ & $\mathrm{n} / \mathrm{a}$ & $\mathrm{n} / \mathrm{a}$ & $\mathrm{n} / \mathrm{a}$ & $\mathrm{n} / \mathrm{a}$ \\
\hline 16 & 0.1 & $>2 / 3$ systemic & None & 0.2 & 57 & 63 & 7 & 12.0 & 4 & 3.4 & 1.2 & 19 & 6 & 4.4 & 5 & 2.9 & 1.0 & $\mathrm{~N}$ \\
\hline 17 & 12 & Suprasystemic & PDA & 13.0 & 55 & 57 & 5 & 13.1 & 2 & 3.8 & 1.0 & 48 & 7 & 9.7 & 2 & 4.2 & 1.0 & $\mathrm{~N}$ \\
\hline 18 & 7 & $\begin{array}{c}\text { Suprasystemic } \\
\text { (120) }\end{array}$ & ASD & $\mathrm{n} / \mathrm{a}$ & $\mathrm{n} / \mathrm{a}$ & $\mathrm{n} / \mathrm{a}$ & $\mathrm{n} / \mathrm{a}$ & $\mathrm{n} / \mathrm{a}$ & $\mathrm{n} / \mathrm{a}$ & $\mathrm{n} / \mathrm{a}$ & $\mathrm{n} / \mathrm{a}$ & $\mathrm{n} / \mathrm{a}$ & $\mathrm{n} / \mathrm{a}$ & $\mathrm{n} / \mathrm{a}$ & $\mathrm{n} / \mathrm{a}$ & $\mathrm{n} / \mathrm{a}$ & $\mathrm{n} / \mathrm{a}$ & $\mathrm{n} / \mathrm{a}$ \\
\hline 19 & 10 & $\begin{array}{c}>2 / 3 \text { systemic } \\
\text { (35) }\end{array}$ & PFO & $\mathrm{n} / \mathrm{a}$ & $\mathrm{n} / \mathrm{a}$ & $\mathrm{n} / \mathrm{a}$ & $\mathrm{n} / \mathrm{a}$ & $\mathrm{n} / \mathrm{a}$ & $\mathrm{n} / \mathrm{a}$ & $\mathrm{n} / \mathrm{a}$ & $\mathrm{n} / \mathrm{a}$ & $\mathrm{n} / \mathrm{a}$ & $\mathrm{n} / \mathrm{a}$ & $\mathrm{n} / \mathrm{a}$ & $\mathrm{n} / \mathrm{a}$ & $\mathrm{n} / \mathrm{a}$ & $\mathrm{n} / \mathrm{a}$ & $\mathrm{n} / \mathrm{a}$ \\
\hline Median & 1.5 & & & 1.5 & 63.0 & 60.0 & 7.8 & 16.5 & 5.0 & 3.1 & 1.0 & 48.0 & 7 & 9.7 & 5 & 2.9 & 1 & \\
\hline IQR & 0.2 & & & 0.4 & 50.7 & 57.5 & 7.0 & 10.7 & 4.0 & 2.5 & 0.8 & 24.7 & 6.7 & 4.5 & 5 & 2.6 & 0.9 & \\
\hline
\end{tabular}

$\mathrm{O}_{2}$ : oxygen; iNO: inhaled nitric oxide; RVSP: right ventricle systolic pressure; mPAP: mean pulmonary artery pressure; MSP: main systemic pressure; PCWP: post-capillary wedge pressure; PVRI: pulmonary vascular resistance index; RA: right atrial pressure; Cl: cardiac index; Qp/Qs: pulmonary-to-systemic blood flow ratio; n/a: not available; IQR: interquartile range; ASD: atrial septal defect; PDA: patent ductus arteriosus beyond neonatal period; VSD: ventricular septal defect; PFO: patent foramen ovale. \#: maximum vasodilation testing was performed by exposure to fraction of insipired $\mathrm{O}_{2} 100 \%$ and iNO $40 \mathrm{ppm}$; responsiveness was defined as a decrease in mPAP of at least $10 \mathrm{mmHg}$ to achieve values <40 $\mathrm{mmHg}$ with a

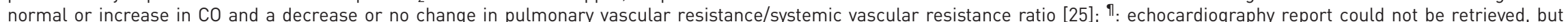
mild pulmonary hypertension was reported in the medical record, prior to PDA closure at age 5 . The patient was subsequently lost to follow-up and untreated until age 18 , when right heart catheterisation revealed very elevated mPAP and pulmonary vascular resistance with intracardiac right-to-left shunting. 

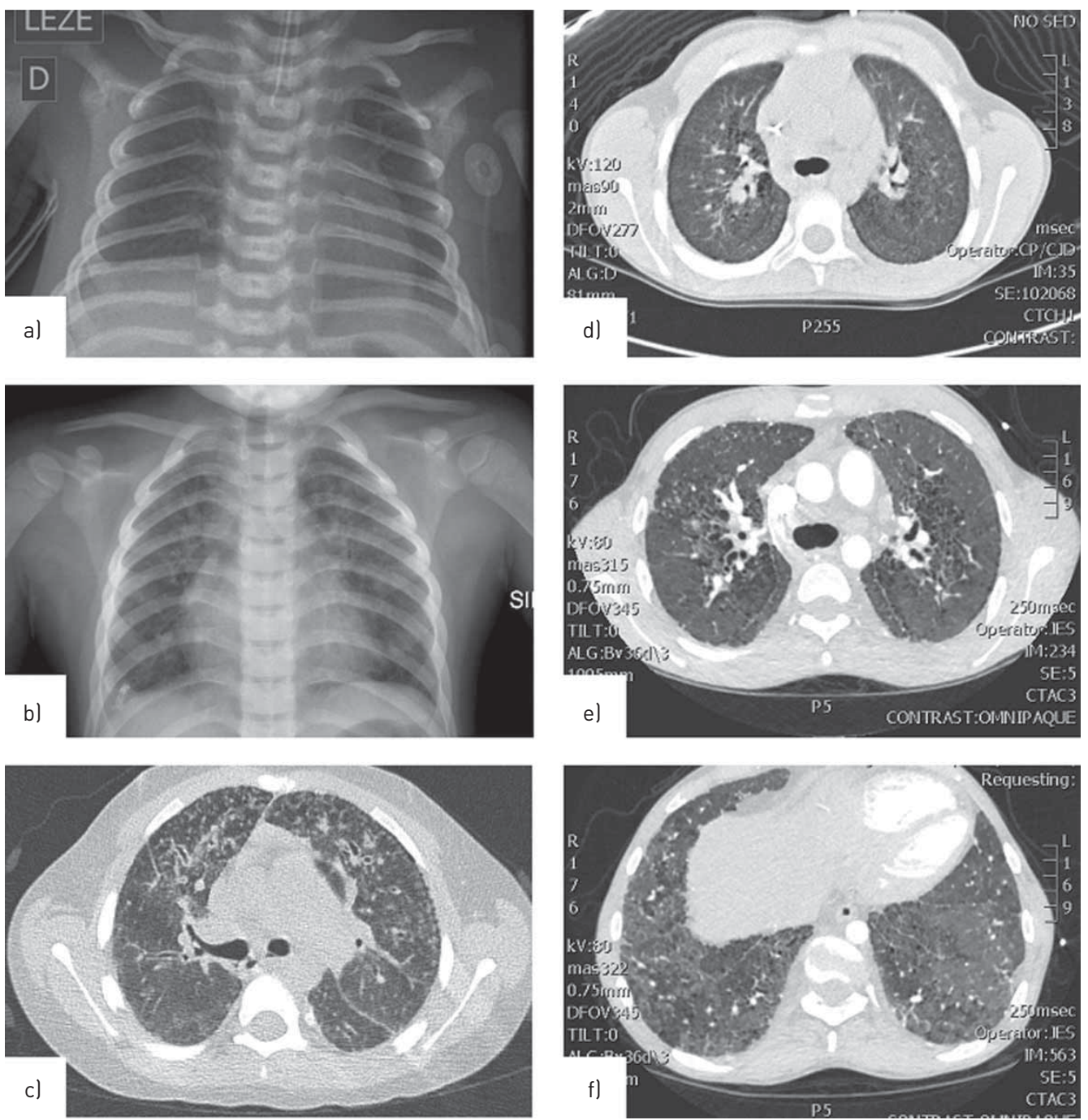

FIGURE 2 Lung imaging. a) Case 1: chest radiography on day 1 with hyperlucent, hypoplastic lungs with poor vascular markings. b, c) Case 19: chest radiography at age 3 years showing ground-glass opacities and bronchial thickening (b); chest computed tomography (CT) at age 10 years showing septal and bronchial wall thickening, honeycombing and diffuse nodules (c). $d$-f) Case 10: chest CTs at d) age 8 years showing diffuse ground-glass opacity and septal thickening, enlarged pulmonary arteries and scattered emphysema; and e, f) age 18 years showing markedly increased interstitial fibrosis with areas of central mosaic density in upper and lower lobes, small cystic areas with some bronchiectasis, some areas of lungs appearing less involved and a parenchymal bulla present in the lingula.

maldevelopment. Evolving interstitial thickening with fibrosis, IBA recruitment and development of interstitial capillary proliferation were also noted.

\section{Discussion}

TBX4 variant carriers are at risk for abnormal distal lung development, PPHN, paediatric-onset $\mathrm{PH}$, multiple congenital anomalies including congenital heart defects and a typical foot malformation, and developmental disabilities. The majority of our patients (63\%) presented with a biphasic clinical course consisting of PPHN and neonatal respiratory failure with apparent resolution around 1 month of age, followed by chronic PH later in infancy or early childhood. It is notable that this form of PH fits a precapillary phenotype; however, given the degree of concurrent lung irregularities, these individuals would not technically meet traditional criteria for World Health Organization Classification Group $1 \mathrm{PH}$ (PAH), and might well be classified as Group 3 (PH due to chronic lung disease and/or hypoxia) [6]. Our description of developmental lung disease in patients with TBX4 variants suggests that associated PH may have several causal associations including chronic respiratory disease and hypoxia in addition to idiopathic $\mathrm{PH}$. Given the difficulty of defining these aetiologies in our retrospective series, we are using the term $\mathrm{PH}$ (versus $\mathrm{PAH}$ ) for TBX4-associated vascular disease. 


\begin{tabular}{|c|c|c|c|c|c|c|c|c|c|c|}
\hline Case & $\begin{array}{l}\text { Age at } \\
\text { biopsy }\end{array}$ & Outcome & $\begin{array}{c}\text { Alveolar } \\
\text { simplification }\end{array}$ & $\begin{array}{c}\text { Wide } \\
\text { interstitium }\end{array}$ & $\begin{array}{l}\text { PIG-like } \\
\text { cells }\end{array}$ & $\begin{array}{l}\text { Interstitial } \\
\text { fibrosis }^{\#}\end{array}$ & $\begin{array}{l}\text { Back-to-back } \\
\text { bronchioli" }\end{array}$ & $\begin{array}{c}\text { Thickened PA } \\
\text { muscular } \\
\text { wall }^{+}\end{array}$ & $\begin{array}{l}\text { PA fibrointimal } \\
\text { proliferation }\end{array}$ & $\begin{array}{l}\text { Intrapulmonary } \\
\text { bronchopulmonary } \\
\text { anastomosis and bronchial } \\
\text { vessel recruitment }\end{array}$ \\
\hline $1 \S$ & 4 months & $\begin{array}{l}\text { Death at } \\
5 \text { months }\end{array}$ & Diffuse & Diffuse & Diffuse & None & Focal & Moderate & Absent & Absent \\
\hline $7^{\S}$ & 3 months & $\begin{array}{l}\text { Death at } \\
8 \text { months }\end{array}$ & Diffuse & Diffuse & Patchy & Patchy & Absent & Moderate & Moderate & Absent \\
\hline $12^{\S}$ & 2 months & $\begin{array}{l}\text { Lung } \\
\text { transplant at } \\
11 \text { years }\end{array}$ & Diffuse & Patchy & Absent & Patchy & Multifocal & Moderate & Absent & Present \\
\hline $13^{\S}$ & 1.5 months & $\begin{array}{c}\text { Severe ILD at } \\
4 \text { years }\end{array}$ & Diffuse & Patchy & Diffuse & Patchy & Absent & Moderate & Absent & Absent \\
\hline $10 a^{f \# \#}$ & 2 years & $\begin{array}{l}\text { Lung } \\
\text { transplant at } \\
18 \text { years }\end{array}$ & Diffuse & Absent & Absent & Absent & Absent & Moderate & Absent & Present \\
\hline $10 b^{f \# \#}$ & 2 years & $\begin{array}{l}\text { Lung } \\
\text { transplant at } \\
18 \text { years }\end{array}$ & Diffuse & Diffuse & Absent & Diffuse & Multifocal & Moderate & Absent & Present \\
\hline $18^{f}$ & 7 years & $\begin{array}{c}\text { Moderate } \mathrm{PH} \\
\text { at } 9 \text { years }\end{array}$ & Diffuse & Diffuse & Absent & Patchy & Absent & Moderate & Absent & Present \\
\hline $19^{f}$ & 7 years & $\begin{array}{l}\text { Moderate } \mathrm{PH} \\
\text { at } 10 \text { years }\end{array}$ & Diffuse & Absent & Absent & Absent & Absent & Moderate & Moderate & Present \\
\hline \multicolumn{11}{|c|}{ 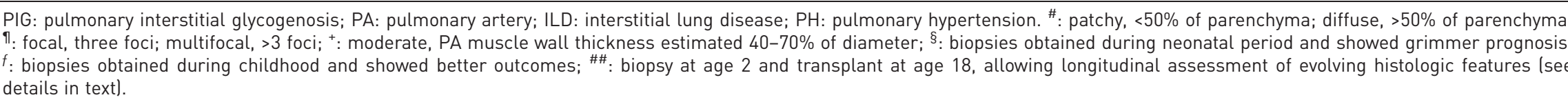 } \\
\hline
\end{tabular}




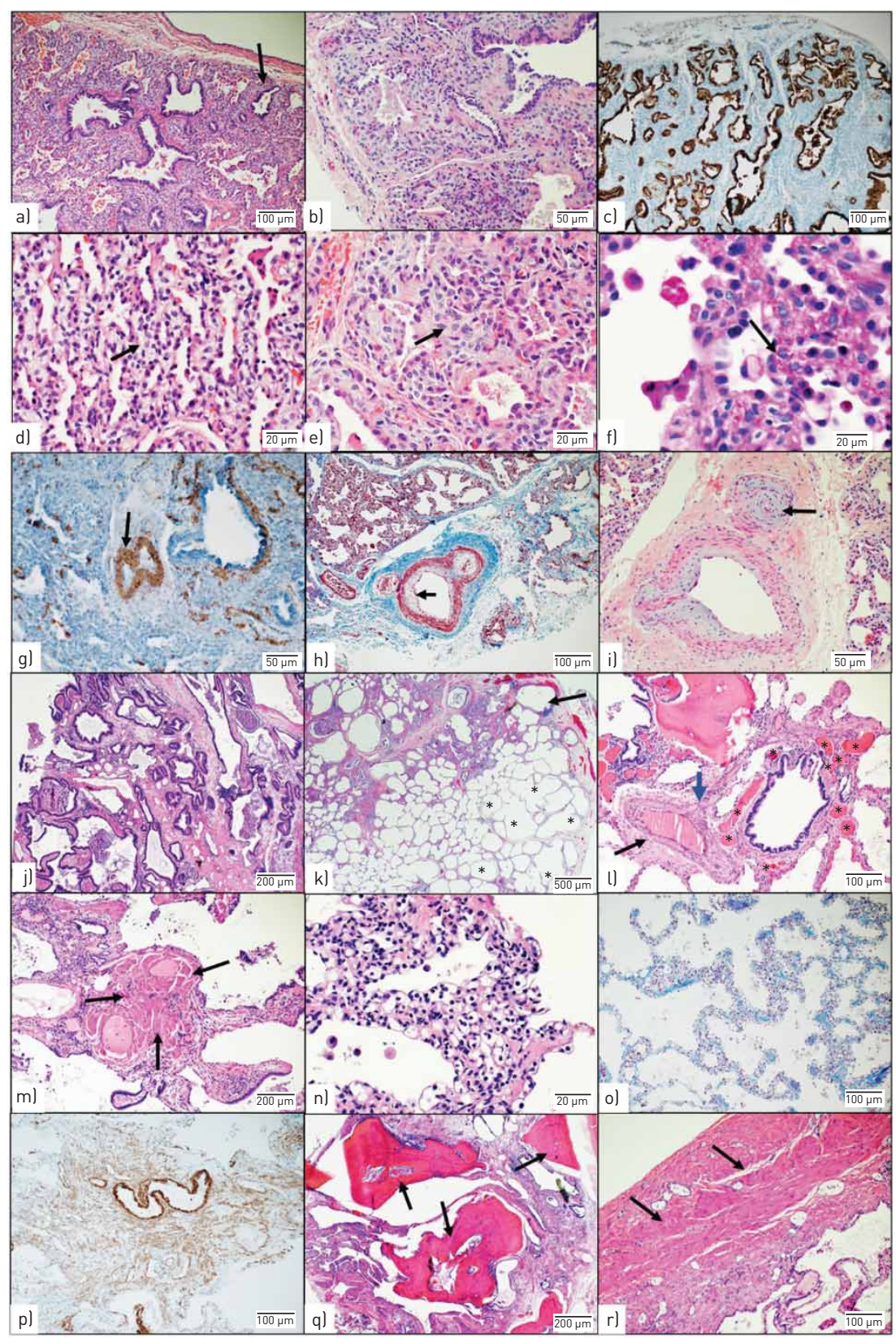

FIGURE 3 Representative histopathology images. Early biopsies obtained in the neonatal period show markedly underdeveloped respiratory lobules $(a, b$; hematoxylin and eosin $(H+E)$ stain), with small rounded immature alveoli, some with canalicular and others with saccular shape (c; cytokeratin stain). A terminal bronchiole reaches the septum (arrow on a), indicating markedly delayed alveolar development. The interstitium is widened by the presence of immature, pale mesenchymal cells (arrows on $d, e ; H+E$ stain); these cells are PAS positive (arrow on f; PAS stain), resembling the features of those of pulmonary interstitial glycogenosis cells. Pulmonary hypertensive remodelling of pulmonary arteries with muscular hypertrophy larrow on g; smooth muscle actin stain) along with fibrointimal proliferation (arrow on h; trichrome stain) (i; $\mathrm{H}$ + E stain) evolving to complete pulmonary arterial obstruction (arrow on i) is identified. Samples from childhood $(j-r)$ showed lack of alveolar development evidenced by multiple foci of back-to-back bronchiolar profile ( $j ; \mathrm{H}+\mathrm{E}$ stain), and a terminal bronchiole extending to the pleura surface (arrow on $\mathrm{k} ; \mathrm{H}+\mathrm{E}$ stain). There were diffuse alveolar growth abnormalities characterised by large, dilated and simplified alveoli lexamples are labelled with asterisks on kl. Marked vascular changes included pulmonary arterial hypertensive remodelling (black arrow on $\mathrm{l} ; \mathrm{H}+\mathrm{E}$ stain); recruitment of bronchial vasculature including intrapulmonary bronchopulmonary anastomosis (blue arrow on (), bronchial veins and microvessels (asterisks on (); and marked muscular hypertrophy of lymphatic vessels (arrows on $\mathrm{m} ; \mathrm{H}+\mathrm{E}$ stain). Pathologic remodelling of the interstitium is seen with the presence of proliferating capillaries ( $n$; $\mathrm{H}+\mathrm{E}$ stain), fibrosis (o; trichrome stain), focal smooth muscle proliferation ( $p$; smooth muscle actin stain) and multiple areas of bone formation larrows on $\mathrm{q} ; \mathrm{H}+\mathrm{E}$ stain). In one case (10b) marked pleural smooth muscle muscularisation is noted larrows on $r$; $\mathrm{H}+\mathrm{E}$ stain). 
Diffuse developmental lung disorders are rare diseases related to aberrations in primary mechanisms of lung airway and vascular development, and include such diagnoses as AD, congenital alveolar dysplasia and alveolar capillary dysplasia with misalignment of the pulmonary veins (ACDMPV), a lethal neonatal disease associated with FOXF1 variants [28]. Emerging evidence shows that developmental lung disorders are phenotypically heterogeneous. ACDMPV was recently reported in older infants with seemingly precapillary PH, suggesting that FOXF1-related disease has a broader clinical spectrum than initially thought $[29,30]$. TBX4 variants were reported in a neonate presenting with lethal $\mathrm{AD}$ [15], one with lethal congenital alveolar dysplasia and one with an undefined alveolar growth abnormality and survival beyond 8 months of age [16]. Our pathology findings, with a broader range of age and clinical manifestations, shed light on the pathogenesis of $\mathrm{PH}$ in TBX4 mutants, even though we cannot exclude a selection bias because the biopsies were obtained on a clinical basis without unified criteria. This study confirms that various developmental abnormalities affecting alveolar, interstitial and vascular structures underlie TBX4-associated PH. These features imply compromised growth of pulmonary endoderm severely affecting airway/alveolar development, and mesenchymal maldevelopment, reflected by hypertensive remodelling of pulmonary arteries, prominent IBA and other findings of interstitial disease. Our longitudinal observation suggests that these pathological fetal processes continue after birth and progress with age in certain cases, with gradual vascular and lymphatic remodelling and development of the collateral circulation, leading to progressive $\mathrm{PH}$ and end-stage lung disease in childhood or young adulthood.

Imaging studies suggest a combination of lung hypoplasia and alveolar dysfunction associated with the neonatal presentation, and progressive bronchial and interstitial changes developing over time in certain cases. The combination of prominent interstitial lung disease and $\mathrm{PH}$ in a subset of patients (cases 2, 12, 13, 18 and 19) suggests that mechanisms related to the lung disease, perhaps including chronic hypoxia, may be a contributing factor. However, RHC data suggest severe pulmonary vascular disease in most cases regardless of parenchymal disease, with earlier age at diagnosis and more severe functional values compared to a reference paediatric PAH cohort (the REVEAL cohort [31]). Lack of response to vasoreactivity testing has also been described in children with BMPR2 mutations compared to those without [32], suggesting that genetic forms of $\mathrm{PH}$ have distinct vascular pathophysiology.

KeRSTJENS-Frederikse et al. [10] first described TBX4 mutations in 30\% of a cohort of children diagnosed with idiopathic/familial PAH and SPS, as opposed to only $2.5 \%$ of a control adult cohort. ZHU et al. [11] calculated a 7.7\% prevalence of TBX4-related disease in a larger cohort of paediatric PAH. LEVY et al. [12] also estimated a 7.5\% TBX4 mutation prevalence in 3 out of 40 infants with PAH. EYRIEs et al. [8] found a TBX4 variant prevalence of one out of $36(2.8 \%)$ and four out of $168(2.4 \%)$ in French children and adults with $\mathrm{PAH}$, respectively, which was lower than the prevalence of a BMPR2 variant of $19.4 \%$ and $14.3 \%$, respectively, in that cohort. A lower frequency of TBX4 variants has been detected in adult-onset PAH than in paediatric-onset $\mathrm{PAH}$, with an overall mutation frequency estimated at $1.5 \%$ (25 out of 1633 cases) $[8,10,11,33,34]$. Overall, the lack of standardised inclusion and diagnostic criteria among centres in this paediatric series precludes any inference on prevalence or comparison with $B M P$-related and other $\mathrm{PAH}$ genes.

TBX4 variants are associated with multiple anomalies, consistent with disruption of key developmental processes beyond the lung. Not all phenotypic features have the same expressivity. Whereas SPS has a high penetrance, that of $\mathrm{PH}$ appears lower [10]. This selective penetrance may putatively depend on the variant itself and its effect on protein dosage and function. However, phenotypical heterogeneity between relatives with a common variant, some having SPS alone and others having a combination of SPS and PH, suggests that TBX4-related $\mathrm{PH}$ is not purely monogenic, and that multiple innate and environmental factors may be at play, similarly to what is observed in other genetic forms of PH [6]. In BMPR2-related disease, PAH penetrance is only $20 \%$, and secondary factors modulate expressivity and disease progression [6]. We observed a 2:1 female prevalence, presumably attributable to selective wastage of male fetuses or an abnormal primary sex ratio. Such disparity was observed in adult $\mathrm{PH}$ prior to the identification of causative genes [35], and confirmed in large registries [36]. The role of sex-dependent hormonal factors [37] and modifier genes [38] was subsequently demonstrated in BMPR2-related PAH, accounting for female predominance. Putatively, similar mechanisms also exist for TBX4.

The severity of $\mathrm{PH}$ does not necessarily correlate with the predicted level of protein expression, as observed in FOXF1-related ACDMPV [39]. Some CNV cases with complete TBX4 haploinsufficiency have less severe $\mathrm{PH}$ than others with less gene-disruptive SNVs in our and other series [10], suggesting either a dominant-negative effect or interactions with genetic or environmental factors, which makes genetic-based prognosis challenging.

There was a greater incidence of developmental disability among our patients with CNVs (cases 1-6) than those with SNVs (cases 7-19), suggesting a role for neighbouring genes, although postnatal factors such as 
PPHN or ECMO cannot be excluded in the CNV group. Comparing our cases with previously published 17q22-q23.2 deletions (supplementary table S3), deletions involving the contiguous TBX2 and TBX4 loci more frequently resulted in $\mathrm{PH}$ and congenital heart defects than did those sparing these two genes, suggesting a major role for these two genes in the cardiovascular components of the syndrome, whereas developmental disability had a homogeneous prevalence regardless of TBX2/4 involvement, suggesting again a role for other neighbouring genes.

TBX2 contributes to airway growth and branching [40] and to endocardial cushion formation, critical in the pathogenesis of septal defects [41]. TBX2 missense mutations were identified in individuals with cardiac septal defects, developmental delays and skeletal anomalies, but no PH [42]. We can speculate that, in the complex pathogenesis of congenital heart defect, TBX4 and TBX2 play significant yet distinct roles as causative or modifier genes, with TBX4 contributing to $\mathrm{PH}$ onset and severity in this disease group. Conversely, developmental delay, hearing loss and skeletal defects were equally represented independently of TBX2/TBX4 involvement, suggesting multiple gene interactions in the pathogenesis of non-cardiovascular anomalies.

Although TBX4 was initially identified as a critical actor in hind limb development [43], it is highly expressed in developing lung mesenchyme [44], with a highly conserved TBX4 enhancer sequence regulating its spatiotemporal expression [45]. Homozygous TBX4 mutant mouse embryos die at embryonic day 10.5 from defective allantois formation and placental insufficiency [46], and conditional lung mesenchymal TBX4 reduction leads to impaired lung development [44]. TBX4 interacts with fibroblast growth factor 10 (FGF10), an essential regulator of the limb and lung bud growth and airway branching [47], which may account for combined lower limb/pulmonary phenotype in TBX4-associated disease. The FGF10 pathway also regulates epithelial expression of thyroid transcription factor 1 (TTF1, encoded by the NKX2.1 gene), a key factor in alveolar development and surfactant synthesis [48], which may contribute to neonatal respiratory symptoms in TBX4 mutants.

Limitations of this study include a recruitment bias towards paediatric and neonatal forms of TBX4-linked $\mathrm{PH}$; a lack of standardised inclusion criteria that precludes estimating the prevalence of TBX4 variants among infants affected with PPHN, infantile/paediatric PH and congenital heart defects; and variable timing of follow-up precluding outcome comparisons.

In summary, this study confirms that TBX4 variants underlie a complex variety of developmental lung disorders, resulting in a spectrum of clinical manifestations including PPHN, neonatal hypoxic respiratory failure, interstitial lung disease and chronic/progressive paediatric $\mathrm{PH}$, often associated with multisystem anomalies. The variability and complexity of the phenotype and its potential overlap with other $\mathrm{PH}$-associated gene defects warrant thorough molecular genetic testing, involving TBX4-inclusive diagnostic panels combined with CNV microarrays, in order to capture both small and large variants. The biphasic evolution we describe, characterised by hypoxic respiratory failure at birth followed by later-onset $\mathrm{PH}$, suggests that infants with severe PPHN, especially if idiopathic, should undergo an appropriate echocardiography follow-up during infancy and early childhood, and should be tested for TBX4 variants when positive and/or in the presence of suggestive features such as congenital heart defects, foot anomalies and SPS. Larger cohort- and population-based studies are needed to better delineate genotype-phenotype correlations and determine future diagnostic and therapeutic strategies.

Author contributions: O. Danhaive and S.H. Abman designed the study and supervised the project; O. Danhaive, C. Galambos, E.D. Austin and M.P. Mullen co-wrote the manuscript; C. Galambos performed the pathology studies in collaboration with J. Johnson and R. Boldrini; J.T. Shieh performed the genetic analysis with the contribution of C. Surace, D. Peca, P.B. Agrawal, M.W. Pauciulo and W.C. Nichols; M.P. Mullen and D. Ivy contributed to the cardiology and haemodynamic analysis; E.D. Austin, M.J. Kielt, M. Griese and S.H. Abman contributed to the pulmonology analysis; N. Schwerk, N. Ullmann, R. Cutrera, I. Stucin-Gantar, C. Haass and M. Bansal contributed to clinical data.

Conflict of interest: C. Galambos has nothing to disclose. M.P. Mullen has acted as a site principal investigator on trials sponsored by United Therapeutics, Actelion, Ikaria and GSK, and received travel support from Actelion, outside the submitted work. J.T. Shieh has nothing to disclose. N. Schwerk has nothing to disclose. M.J. Kielt has nothing to disclose. N. Ullmann has nothing to disclose. R. Boldrini has nothing to disclose. I. Stucin-Gantar has nothing to disclose. C. Haass has nothing to disclose. M. Bansal has nothing to disclose. P.B. Agrawal has nothing to disclose. J. Johnson has nothing to disclose. D. Peca has nothing to disclose. C. Surace has nothing to disclose. R. Cutrera has nothing to disclose. M.W. Pauciulo has nothing to disclose. W.C. Nichols has nothing to disclose. M. Griese has nothing to disclose. D. Ivy has contracts (through the University of Colorado School of Medicine) with Actelion, Bayer, Lilly and United Therapeutics for consultancy and research studies. S.H. Abman has nothing to disclose. E.D. Austin has nothing to disclose. O. Danhaive has nothing to disclose.

Support statement: This publication was supported in part by the Frederick and Margaret L. Weyerhaeuser Foundation, the Jayden de Luca Foundation (D. Ivy, C. Galambos), NIH grants R01HL114753 and U01HL121518 (S.H. Abman), NIH/NCATS Colorado CTSA grant number UL1 TR002535, NIH grant HL105333 (W.C. Nichols, M.W. Pauciulo), and 
an unrestricted grant from the Chiesi Foundation, Parma, Italy (O. Danhaive, R. Cutrera, D. Peca). Funding information for this article has been deposited with the Crossref Funder Registry.

\section{References}

1 van Loon RL, Roofthooft MT, Hillege HL, et al. Pediatric pulmonary hypertension in the Netherlands: epidemiology and characterization during the period 1991 to 2005. Circulation 2011; 124: 1755-1764.

2 Humbert M, Sitbon O, Chaouat A, et al. Pulmonary arterial hypertension in France: results from a national registry. Am J Respir Crit Care Med 2006; 173: 1023-1030.

3 Li L, Jick S, Breitenstein S, et al. Pulmonary arterial hypertension in the USA: an epidemiological study in a large insured pediatric population. Pulm Circ 2017; 7: 126-136.

4 Zijlstra $\mathrm{WMH}$, Douwes JM, Rosenzweig EB, et al. Survival differences in pediatric pulmonary arterial hypertension: clues to a better understanding of outcome and optimal treatment strategies. J Am Coll Cardiol 2014; 63: 2159-2169.

5 Soubrier F, Chung WK, Machado R, et al. Genetics and genomics of pulmonary arterial hypertension. J Am Coll Cardiol 2013; 62: 25 Suppl., D13-D21.

6 Ma L, Chung WK. The role of genetics in pulmonary arterial hypertension. J Pathol 2017; 241: 273-280.

7 Harrison RE, Berger R, Haworth SG, et al. Transforming growth factor- $\beta$ receptor mutations and pulmonary arterial hypertension in childhood. Circulation 2005; 111: 435-441.

8 Eyries M, Montani D, Nadaud S, et al. Widening the landscape of heritable pulmonary hypertension mutations in paediatric and adult cases. Eur Respir J 2019; 53: 1801371.

9 Bongers EM, Duijf PH, van Beersum SE, et al. Mutations in the human TBX4 gene cause small patella syndrome. Am J Hum Genet 2004; 74: 1239-1248.

10 Kerstjens-Frederikse WS, Bongers EM, Roofthooft MT, et al. TBX4 mutations (small patella syndrome) are associated with childhood-onset pulmonary arterial hypertension. J Med Genet 2013; 50: 500-506.

11 Zhu N, Gonzaga-Jauregui C, Welch CL, et al. Exome sequencing in children with pulmonary arterial hypertension demonstrates differences compared with adults. Circ Genom Precis Med 2018; 11: e001887.

12 Levy M, Eyries M, Szezepanski I, et al. Genetic analyses in a cohort of children with pulmonary hypertension. Eur Respir J 2016; 48: 1118-1126.

13 Steurer MA, Jelliffe-Pawlowski LL, Baer RJ, et al. Persistent pulmonary hypertension of the newborn in late preterm and term infants in California. Pediatrics 2017; 139: e20161165.

14 Lazar DA, Olutoye OO, Cass DL, et al. Outcomes of neonates requiring extracorporeal membrane oxygenation for irreversible pulmonary dysplasia: the Extracorporeal Life Support Registry experience. Pediatr Crit Care Med 2012; 13: $188-190$

15 Szafranski P, Coban-Akdemir ZH, Rupps R, et al. Phenotypic expansion of TBX4 mutations to include acinar dysplasia of the lungs. Am J Med Genet A 2016; 170: 2440-2444.

16 Suhrie K, Pajor NM, Ahlfeld SK, et al. Neonatal lung disease associated with TBX4 mutations. J Pediatr 2019; 206: 286-292.

17 Kircher M, Witten DM, Jain P, et al. A general framework for estimating the relative pathogenicity of human genetic variants. Nat Genet 2014; 46: 310-315.

18 Lek M, Karczewski KJ, Minikel EV, et al. Analysis of protein-coding genetic variation in 60,706 humans. Nature 2016; 536: 285-291.

19 Landrum MJ, Lee JM, Benson M, et al. ClinVar: improving access to variant interpretations and supporting evidence. Nucleic Acids Res 2018; 46: D1062-D1067.

20 Rehm HL, Berg JS, Brooks LD, et al. ClinGen-the clinical genome resource. N Engl J Med 2015; 372: 2235-2242.

21 Kearney HM, Thorland EC, Brown KK, et al. American College of Medical Genetics standards and guidelines for interpretation and reporting of postnatal constitutional copy number variants. Genet Med 2011; 13: 680-685.

22 Richards S, Aziz N, Bale S, et al. Standards and guidelines for the interpretation of sequence variants: a joint consensus recommendation of the American College of Medical Genetics and Genomics and the Association for Molecular Pathology. Genet Med 2015; 17: 405-424.

23 Ballif BC, Theisen A, Rosenfeld JA, et al. Identification of a recurrent microdeletion at 17q23.1q23.2 flanked by segmental duplications associated with heart defects and limb abnormalities. Am J Hum Genet 2010; 86: 454-461.

24 Hutcheon JA, Jacobsen GW, Kramer MS, et al. Small size at birth or abnormal intrauterine growth trajectory: which matters more for child growth? Am J Epidemiol 2016; 183: 1107-1113.

25 Abman SH, Hansmann G, Archer SL, et al. Pediatric pulmonary hypertension: guidelines from the American Heart Association and American Thoracic Society. Circulation 2015; 132: 2037-2099.

26 Citti A, Peca D, Petrini S, et al. Ultrastructural characterization of genetic diffuse lung diseases in infants and children: a cohort study and review. Ultrastruct Pathol 2013; 37: 356-365.

27 Langston C. New concepts in the pathology of congenital lung malformations. Semin Pediatr Surg 2003; 12: 17-37.

28 Deutsch GH, Young LR, Deterding RR. Diffuse lung disease in young children: application of a novel classification scheme. Am J Respir Crit Care Med 2007; 176: 1120-1128.

29 Ito Y, Akimoto T, Cho K, et al. A late presenter and long-term survivor of alveolar capillary dysplasia with misalignment of the pulmonary veins. Eur J Pediatr 2015; 174: 1123-1126.

30 Towe CT, White FV, Grady RM, et al. Infants with atypical presentations of alveolar capillary dysplasia with misalignment of the pulmonary veins who underwent bilateral lung transplantation. J Pediatr 2018; 194: 158-164.

31 Barst RJ, McGoon MD, Elliott CG, et al. Survival in childhood pulmonary arterial hypertension: insights from the registry to evaluate early and long-term pulmonary arterial hypertension disease management. Circulation 2012; 125: 113-122.

32 Rosenzweig EB, Morse JH, Knowles JA, et al. Clinical implications of determining BMPR2 mutation status in a large cohort of children and adults with pulmonary arterial hypertension. J Heart Lung Transplant 2008; 27: 668-674.

33 Navas P, Tenorio J, Quezada CA, et al. Molecular analysis of BMPR2, TBX4, and KCNK3 and genotype-phenotype correlations in Spanish patients and families with idiopathic and hereditary pulmonary arterial hypertension. Rev Esp Cardiol (Engl Ed) 2016; 69: 1011-1019. 
34 Graf S, Haimel M, Bleda M, et al. Identification of rare sequence variation underlying heritable pulmonary arterial hypertension. Nat Commun 2018; 9: 1416.

35 Loyd JE, Butler MG, Foroud TM, et al. Genetic anticipation and abnormal gender ratio at birth in familial primary pulmonary hypertension. Am J Respir Crit Care Med 1995; 152: 93-97.

36 Fessel JP, Loyd JE, Austin ED. The genetics of pulmonary arterial hypertension in the post-BMPR2 era. Pulm Circ 2011; 1: 305-319.

37 Austin ED, Cogan JD, West JD, et al. Alterations in oestrogen metabolism: implications for higher penetrance of familial pulmonary arterial hypertension in females. Eur Respir J 2009; 34: 1093-1099.

38 Yan L, Cogan JD, Hedges LK, et al. The Y chromosome regulates BMPR2 expression via SRY: a possible reason "why" fewer males develop pulmonary arterial hypertension. Am J Respir Crit Care Med 2018; 198: 1581-1583.

39 Dharmadhikari AV, Szafranski P, Kalinichenko VV, et al. Genomic and epigenetic complexity of the FOXF1 locus in 16q24.1: implications for development and disease. Curr Genomics 2015; 16: 107-116.

40 Ludtke TH, Farin HF, Rudat C, et al. Tbx2 controls lung growth by direct repression of the cell cycle inhibitor genes Cdkn1a and Cdkn1b. PLoS Genet 2013; 9: e1003189.

41 Harrelson Z, Kelly RG, Goldin SN, et al. Tbx2 is essential for patterning the atrioventricular canal and for morphogenesis of the outflow tract during heart development. Development 2004; 131: 5041-5052.

42 Liu N, Schoch K, Luo X, et al. Functional variants in TBX2 are associated with a syndromic cardiovascular and skeletal developmental disorder. Hum Mol Genet 2018; 27: 2454-2465.

43 Arora R, Metzger RJ, Papaioannou VE. Multiple roles and interactions of Tbx4 and Tbx5 in development of the respiratory system. PLoS Genet 2012; 8: e1002866.

44 Xie T, Liang J, Liu N, et al. Transcription factor TBX4 regulates myofibroblast accumulation and lung fibrosis. $J$ Clin Invest 2016; 126: 3063-3079.

45 Zhang W, Menke DB, Jiang M, et al. Spatial-temporal targeting of lung-specific mesenchyme by a Tbx4 enhancer. BMC Biol 2013; 11: 111 .

46 Naiche LA, Arora R, Kania A, et al. Identity and fate of Tbx4-expressing cells reveal developmental cell fate decisions in the allantois, limb, and external genitalia. Dev Dyn 2011; 240: 2290-2300.

47 Sekine K, Ohuchi H, Fujiwara M, et al. Fgf10 is essential for limb and lung formation. Nat Genet 1999; 21: 138-141.

48 Peca D, Petrini S, Tzialla C, et al. Altered surfactant homeostasis and recurrent respiratory failure secondary to TTF-1 nuclear targeting defect. Respir Res 2011; 12: 115.

49 Shetty R, Joshi D, Jain M, et al. Rudhira/BCAS3 is essential for mouse development and cardiovascular patterning. Sci Rep 2018; 8: 5632.

50 Jansen S, Geuer S, Pfundt R, et al. De novo truncating mutations in the last and penultimate exons of PPM1D cause an intellectual disability syndrome. Am J Hum Genet 2017; 100: 650-658.

51 Kent WJ, Sugnet CW, Furey TS, et al. The human genome browser at UCSC. Genome Res 2002; 12: 996-1006.

52 Keller RL, Tacy TA, Hendricks-Munoz K, et al. Congenital diaphragmatic hernia: endothelin-1, pulmonary hypertension, and disease severity. Am J Respir Crit Care Med 2010; 18: 555-561.

53 Kurland G, Deterding RR, Hagood JS, et al. An official American Thoracic Society clinical practice guideline: classification, evaluation, and management of childhood interstitial lung disease in infancy. Am J Respir Crit Care Med 2013; 188: 376-394. 